UC-66b

Issued: April 1982

LA- -9222-HDR

DE82 015898

\title{
Heat-Flow Reconnaissance of the Gulf Coastal Plain
}

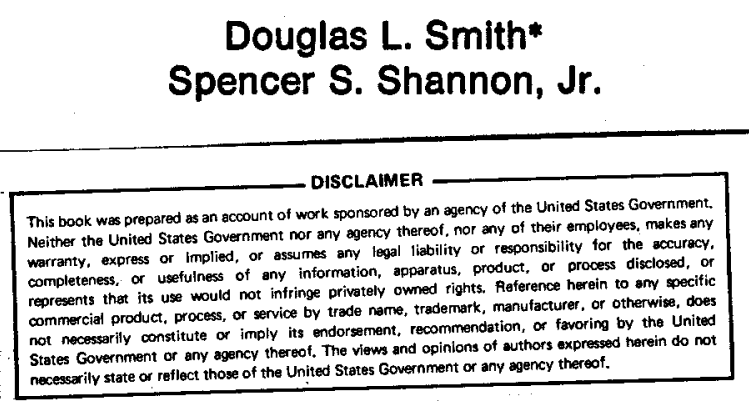

*Department of Geology, University of Florida, Gainesville, FL 32611.

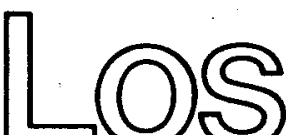
Los Alamos,New Mexico 87545 



\section{DISCLAIMER}

This report was prepared as an account of work sponsored by an agency of the United States Government. Neither the United States Government nor any agency Thereof, nor any of their employees, makes any warranty, express or implied, or assumes any legal liability or responsibility for the accuracy, completeness, or usefulness of any information, apparatus, product, or process disclosed, or represents that its use would not infringe privately owned rights. Reference herein to any specific commercial product, process, or service by trade name, trademark, manufacturer, or otherwise does not necessarily constitute or imply its endorsement, recommendation, or favoring by the United States Government or any agency thereof. The views and opinions of authors expressed herein do not necessarily state or reflect those of the United States Government or any agency thereof. 


\section{DISCLAIMER}

Portions of this document may be illegible in electronic image products. Images are produced from the best available original document. 
HEAT-FLOW RECONNAISSANCE OF THE GULF COASTAL PLAIN

by

Douglas L. Smith and Spencer S. Shannon, Jr.

\section{ABSTRACT}

Most of the 46 new values of heat flow determined for the Gulf Coastal Plain are in the low to normal range, but heat-flow values averaging 1.8 heat-flow unit (HFU) were obtained in Claiborne, Ouachita, and Union parishes, Louisiana. Moreover, a zone of relatively high heat-flow values and steep thermal gradients $\left(35-46^{\circ} \mathrm{C} / \mathrm{km}\right)$ extends from northern Louisiana into southwestern Mississippi. Also near Pensacola, Florida, temperatures of $50^{\circ} \mathrm{C}$ at $1-\mathrm{km}$ depth have been extrapolated from thermal gradients. Future development of low-grade geothermal resources may be warranted in these areas.

\section{INTRODUCTION}

This report presents the results and interpretations derived from a project to acquire additional temperature-gradient and heat-flow data for a reconnaissance of geothermal conditions in the southeastern United States. Temperatures were measured in available boreholes, selected through cooperation with state geological surveys and the United States Geological Survey Water Resources Division.

In the summer of 1979,21 new heat-flow values were obtained from wells in Florida, Alabama, Mississippi, and Louisiana. The range of values suggested a westerly extension of the low-to-normal heat flux, which characterizes the southern Atlantic Coastal Plain and the southern Appalachian Mountains (Smith, Gregory, and Emhof, 1981)! No exceptionally high anomalies were found, but the areas having higher values in northern Louisiana and southwestern Mississippi should be investigated. Anomalous high values 
reported in Arkansas (Fig. 1) by von Frese et al: (1980) support the credibility of the Louisiana values. Similarly, the initial work seems to extend a zone of anomalously low heat flux into central Alabama from the Appalachian Plateau and Valley and Ridge provinces of northeastern Alabama and northwestern Georgia (Fig. 2).

Thermal gradients exceeding $30^{\circ} \mathrm{C} / \mathrm{km}$ were recorded in several wells, and conditions at other sites suggest that the subsurface-water temperatures approach those of low-grade resources.

Some bias was introduced into the selection of borehole sites for continuation of the project in 1980 because results of the first phase included areas having higher heat-flow values than expected, and these required further investigation. Examination of other areas was necessary to achieve a relatively uniform spatial distribution of data points throughout the Gulf coastal plain. Twenty-five new heat-flow values were determined in 1980. Measurements were not made in certain areas, such as southern Louisiana, because no nonflowing boreholes were available.

All 46 values determined in 1979-80, and the procedures for the acquisition of these data, are presented herein. The resource potential of favorable areas, and their relations to basement structure are interpreted from the data acquired.

\section{PROCEDURES}

Borehole temperatures were measured at discrete depths with a thermistor-probe assembly coupled to a four-conductor cable. A Mueller-type Wheatstone bridge having an Electroscientific Industries six-decade variable resistor and a Leeds and Northrup 2437 null detector was used to match thermistor resistances. The system was calibrated from $10^{\circ} \mathrm{C}$ to $40^{\circ} \mathrm{C}$ using a platinum resistance thermometer. The estimated accuracy of individual temperature measurements is $\pm 0.1^{\circ} \mathrm{C}$. The accuracy of successive-temperature differentials obtained from computer-derived, least-squares gradient values is considered to be $\pm 0.01^{\circ} \mathrm{C} / \mathrm{km}$. The terrain at all drill hole sites is sufficiently gentle that no topographic corrections to the computed-temperature gradients are necessary (Smith and Dees, 1982).

Thermal-conductivity values were determined with two divided-bar assemblies similar to those described by Sass et a1. (1971). Both bars were calibrated with fused-quartz and natural-quartz standards supplied by the 


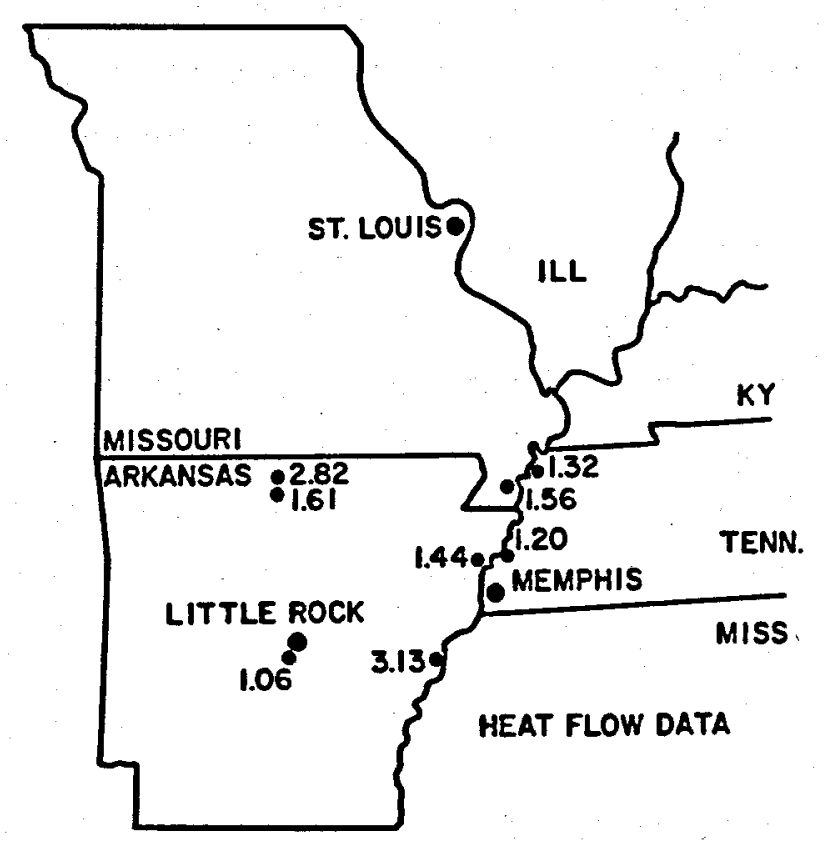

Fig. 1.

Heat-flow data (in heat-flow units). from wells in the Mississippi embayment (from von Frese et al., 1980).

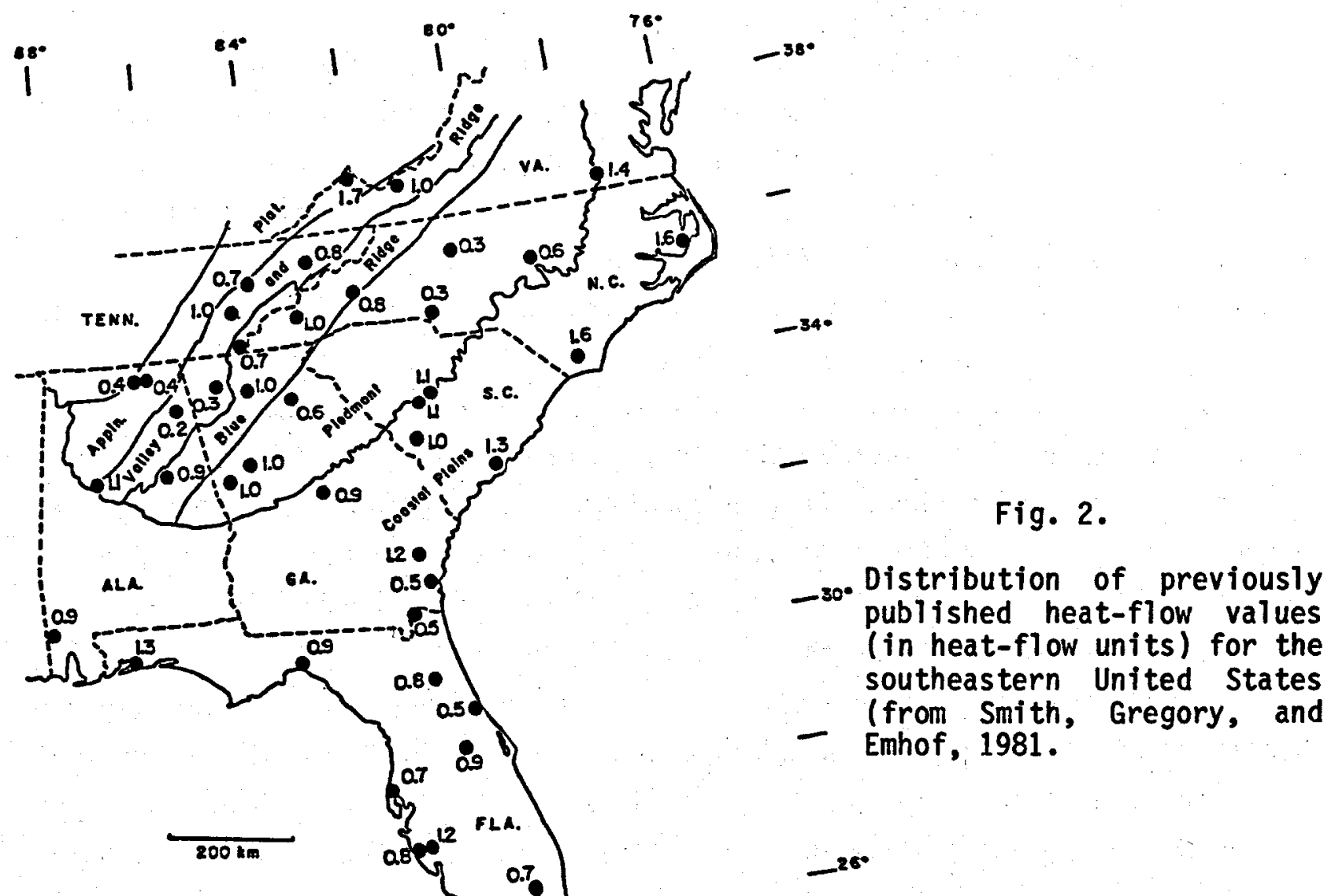


Val tec Corporation having the values of Beck (1957). No boreholes logged had been cored, so only cuttings were available for conductivity measurements. For many sites where rock samples were unavailable, cuttings from nearby drill holes were used.

Thermal-conductivity values on drill cuttings were determined following the procedures and using equipment described by Sass, Lachenbruch, and Munroe (1971). The Coastal Plains sedimentary rocks were assigned a uniform porosity of $30 \%$, but the observed variations in porosity would not affect the calculated conductivity values significantly. Heat-flow values were determined as the product of least-squares temperature gradients and harmonic means of thermalconductivity values.

DATA

The temperature-gradient, thermal-conductivity, and location data for the 46 drill holes are summarized in Table I. Figure 3 depicts the location of new-value measurements except for two sites in north-central Florida beyond the eastern limit of the map.

Temperature gradients commonly vary within a single borehole. Such changes result from lateral flow in aquifers, or rarely from contrasts in strata that have distinctively different thermal-conductivity values. Figures 4 through 49 show the depth/temperature profiles. Measurements at Winnfield, Louisiana, and Columbus, Mississippi, have large changes in gradients. For each borehole, a separate heat flow was computed for each depth zone, and an average value, weighted by depth intervals, was determined and plotted on the figures. The observed variations in some boreholes are examples as to why caution must be used in extrapolating temperatures to greater depths.

A low-to-average heat flow predominates throughout the eastern Gulf Coastal Plain. Except for a limited area in the western part of the Florida panhandle (Fig. 3), all new heat-flow values from southwestern Georgia, Alabama, and Florida are less than 1 heat-flow unit (HFU).

Measurements at Natchez and Utica in southwestern Mississippi yielded thermal gradients as high as $46^{\circ} \mathrm{C} / \mathrm{km}$ (over the 180 - to $340-\mathrm{m}$-depth interval at Utica) and heat-flow values of $1.4 \mathrm{HFU}$ and $1.5 \mathrm{HFU}$ (Fig. 3). Utica overlies the Jackson Dome, a major subsurface feature associated with elevated temperatures discovered from petroleum-exploration efforts. Values from ten sites in 


\section{TABLE I}

\section{GEOTHERMAL-GRADIENT AND HEAT-FLOW DATA}

FOR HOLES LOGGED IN THE GULF COASTAL PLAIN

\begin{tabular}{|c|c|c|c|c|c|c|c|c|c|}
\hline Lecation & Letitude & Longitude & Depth & $\begin{array}{r}\text { Gradtent } \\
\left({ }^{\circ} \mathrm{C} / \mathrm{ka}\right)\end{array}$ & $\begin{array}{c}\text { Interval } \\
(a)\end{array}$ & $\begin{array}{l}\text { No. Sanple } \\
\text { Analyzed }\end{array}$ & $\begin{array}{c}\text { Conductivity } \\
\text { Units } \\
\left(\text { acal/casec }{ }^{*} \mathrm{C}\right)\end{array}$ & $\begin{array}{r}\text { Heat Flo } \\
\text { Units } \\
\text { Incal/ce } \\
\end{array}$ & $\begin{array}{l}\text { on } \\
2 \sec )\end{array}$ \\
\hline \multicolumn{10}{|l|}{ RORIDA } \\
\hline $\begin{array}{l}\text { Gainesville } \\
\text { Deerhaven }\end{array}$ & $29^{\circ} 45^{\prime} 32^{\prime \prime}$ & $82^{\circ} 23^{\prime} 18^{\prime \prime}$ & 500 & 12.04 & $100-500$ & 34 & 3.11 & & 0.37 \\
\hline Mellborn & $30^{\circ} 09^{\prime} 35^{\prime \prime}$ & $82^{\circ} 48^{\prime} 45^{\prime \prime}$ & 300 & 19.71 & $10-300$ & 3 & 3.15 & & 0.62 \\
\hline Gretna & $30^{\circ} 37^{\prime} 13^{\prime \prime}$ & $4^{\prime} 38^{\prime} 24^{\prime \prime}$ & 280 & $\begin{array}{l}26.26 \\
24.82\end{array}$ & $\begin{array}{r}60-160 \\
200-280\end{array}$ & $\frac{6}{2}$ & $\begin{array}{l}3.22 \\
3.40\end{array}$ & $\begin{array}{l}0.84 \\
0.84\end{array}$ & 0.84 \\
\hline St. Ceorge Is. & $29^{\circ} 40^{\prime} 30^{\circ}$ & R'so'05" & 273 & 21.18 & $120-273$ & 10 & 2.77 & & 0.58 \\
\hline $\begin{array}{l}\text { Chattuhoochte } \\
\text { uscs Mell M12493 }\end{array}$ & $30^{\circ} 42^{\prime} 30^{\circ}$ & $84^{\circ} 53^{\prime} 02^{\prime \prime}$ & 140 & 23.77 & $20-140$ & 7 & 3.49 & & 0.83 \\
\hline $\begin{array}{l}\text { Prime Beef } \\
\text { USCS yell M4102 }\end{array}$ & $30^{\circ} 30^{\prime} 21^{\prime \prime}$ & $86^{\circ} 35^{\prime} 14^{\prime \prime}$ & 300 & 27.73 & $40-300$ & 23 & 5.56 & & 1.54 \\
\hline Ft. Malton Beach & $30^{\circ} 23^{\prime} 55^{\prime \prime}$ & $86^{\circ} 35^{\circ} 52^{\prime \prime}$ & 500 & $\begin{array}{l}31.54 \\
23.52\end{array}$ & $\begin{array}{l}100-340 \\
340-500\end{array}$ & 13 & $\begin{array}{l}3.29 \\
2.88\end{array}$ & $\begin{array}{l}1.03 \\
0.67\end{array}$ & 0.898 \\
\hline Maverte & $30^{\circ} 24^{\prime} 09^{\prime}$ & $86^{\circ} 52^{\prime} 35^{\prime \prime}$ & 280 & $\begin{array}{l}22.45 \\
34.13\end{array}$ & $\begin{array}{r}60-140 \\
140-280\end{array}$ & 28 & 3.29 & $\begin{array}{l}0.74 \\
1.12\end{array}$ & 0.988 \\
\hline Cap Henderson & $30^{\circ} 59^{\circ} 42^{\prime \prime}$ & $86^{\circ} 58^{\prime} 100^{\prime \prime}$ & 148 & 12.49 & $60-148$ & 6 & 3.34 & & 0.4 \\
\hline Whiting field & $30^{\circ} 42^{\circ} 52^{\prime \prime}$ & $87^{\circ} 00^{\circ} 22^{\prime \prime}$ & 350 & $\begin{array}{l}28.70 \\
27.81\end{array}$ & $\begin{array}{l}120-260 \\
300-360\end{array}$ & $\frac{6}{3}$ & $\begin{array}{l}3.66 \\
3.52\end{array}$ & $\begin{array}{l}1.05 \\
0.97\end{array}$ & 1.038 \\
\hline \multicolumn{10}{|l|}{ CEORGIA } \\
\hline $\begin{array}{l}\text { Kolomakt } \\
\text { Sute Park }\end{array}$ & $31^{\circ} 25^{\prime} 43^{\circ}$ & $84^{\circ} 57$ '51" & 168 & 19.66 & $60-168$ & 7 & 3.28 & & 0.64 \\
\hline \multicolumn{10}{|l|}{ ALABASA } \\
\hline $\begin{array}{l}\text { Ozark } \\
\text { USES Mell die } 1\end{array}$ & $31^{\bullet} 22^{\prime} 39^{\bullet}$ & $65^{\circ} 34^{\circ} 49^{\prime \prime}$ & 160 & 10.36 & $40-140$ & 8 & 4.75 & & 0.49 \\
\hline Unton Springs & $32^{\circ} 08^{\prime} 09^{\prime}$ & $85^{\circ} 40^{\prime} 54^{\circ}$ & 267 & 23.84 & $80-267$ & $\bullet$ & 3.10 & & 0.74 \\
\hline $\begin{array}{l}\text { Eclectic } \\
\text { usos Mell Ela } 2\end{array}$ & $32^{\bullet} 37^{\prime} 57^{\prime \prime}$ & $85^{\circ} 01^{\prime} 39^{\prime \prime}$ & 120 & 7.08 & $20-110$ & 5 & 4.17 & & 0.29 \\
\hline Thowaston & $32^{\circ} 13^{\prime} 06^{\circ}$ & $87^{\circ} 37^{\prime} 30^{\circ}$ & 340 & $\begin{array}{l}22.20 \\
37.07 \\
19.92\end{array}$ & $\begin{array}{r}10-120 \\
140-280 \\
300-340\end{array}$ & 3 & $\begin{array}{l}2.27 \\
2.38\end{array}$ & $\begin{array}{l}0.50 \\
0.88\end{array}$ & $0.74:$ \\
\hline $\begin{array}{l}\text { Euraw } \\
\text { USCS Mell or } 3\end{array}$ & $32^{\circ} 50^{\prime} 05^{\prime \prime}$ & $87^{\circ} 53^{\prime} 20^{\prime \prime}$ & 108 & 10.70 & $20-108$ & 6 & 4.71 & & 0.50 \\
\hline Gutn & $33^{\circ} 57^{\prime} 42^{\prime}$ & $97^{\circ} 55^{\circ} 00^{\prime \prime}$ & 149 & $\begin{array}{r}0.76 \\
11.28\end{array}$ & $\begin{array}{r}20-120 \\
120-149\end{array}$ & 6 & 3.50 & & 0.39 \\
\hline \multicolumn{10}{|l|}{ MISSISSIPPI } \\
\hline $\begin{array}{l}\text { Columbus } \\
\text { Mooker Chea } 122\end{array}$ & $33^{\circ} 25$ ' $28^{\prime \prime}$ & $28^{\circ} 23^{\prime} 63^{\prime \prime}$ & 306 & $\begin{array}{l}17.31 \\
15.70\end{array}$ & $\begin{array}{r}20-100 \\
100-306\end{array}$ & 3 & 3.55 & $\begin{array}{l}0.62 \\
0.56\end{array}$ & 0.584 \\
\hline Irooklyn & $31^{*} 01^{\prime} 35^{\prime \prime}$ & $89^{\circ} 09^{\prime} 31^{\prime \prime}$ & 218 & 20.08 & $60-220$ & 14 & 5.06 & & 1.06 \\
\hline $\begin{array}{l}\text { Forest } \\
\text { Industrial Prt }\end{array}$ & $32^{\circ} 22^{\prime} 45^{\circ}$ & $89^{\circ} 30^{\prime} 05^{\prime \prime}$ & 380 & $\begin{array}{l}21.09 \\
27.73\end{array}$ & $\begin{array}{l}120-240 \\
240-380\end{array}$ & 20 & 3.77 & $\begin{array}{c}0 \\
0.78 \\
1.06\end{array}$ & $0.92 a$ \\
\hline $\begin{array}{l}\text { Ourant } \\
\text { USCS Nell TH4 }\end{array}$ & $33^{\circ} 04^{\prime} 58^{\prime \prime}$ & $89^{\circ} 51^{\circ} 09^{\circ}$ & 119 & 8.94 & $20-100$ & 7 & 6.15 & & 0.46 \\
\hline Senatoo ia & $34^{\circ} 33^{\prime} 24^{\circ}$ & $89^{\circ} 58^{\prime} 12^{\prime \prime}$ & 334 & 19.94 & $100-340$ & 18 & 5.10 & & 1.01 \\
\hline Utica & $32^{\circ} 06^{\prime} 30^{\prime \prime}$ & $90^{\circ} 37 \cdot 30^{\prime \prime}$ & 380 & $\begin{array}{l}25.75 \\
45.98\end{array}$ & $\begin{array}{r}80-160 \\
180-340\end{array}$ & $\begin{array}{l}5 \\
16\end{array}$ & $\begin{array}{l}6.50 \\
3.09\end{array}$ & $\begin{array}{l}1.67 \\
1.42\end{array}$ & 1.482 \\
\hline $\begin{array}{l}\text { Shelby } \\
\text { USGS vell } 032\end{array}$ & $33^{\circ} 56^{\prime} 30^{\prime}$ & $90^{\circ} 46^{\prime} 15^{\prime \prime}$ & 500 & $\begin{array}{l}49.97 \\
23.97\end{array}$ & $\begin{array}{r}20-80 \\
100-500\end{array}$ & 7 & 4.86 & $\begin{array}{l}2.43 \\
2.16\end{array}$ & $1.33^{\mathrm{a}}$ \\
\hline
\end{tabular}


Table I (cont)

\begin{tabular}{|c|c|c|c|c|c|c|c|c|c|}
\hline Location & Letfude & Longitude & Depth & $\begin{array}{c}\text { Gradient } \\
(\mathrm{c} / \mathrm{km})\end{array}$ & $\begin{array}{c}\text { Interval } \\
\text { (a) }\end{array}$ & $\begin{array}{l}\text { Mo. Sumple } \\
\text { Analyzed }\end{array}$ & $\begin{array}{c}\text { Conduetivity } \\
\text { Units } \\
\left(\operatorname{mal} / \mathrm{cmsec}{ }^{*} \mathrm{c}\right)\end{array}$ & $\begin{array}{l}\text { Heat Flo } \\
\text { Units } \\
\text { Incal/cm? }\end{array}$ & $2 \sec 1$ \\
\hline \multicolumn{10}{|c|}{ mussissippI (continued) } \\
\hline $\begin{array}{l}\text { Pace } \\
\text { USGS Mell Latat3 }\end{array}$ & $33^{\circ} 47^{\prime} 37^{*}$ & $90^{\circ} 51^{\prime} 18^{\circ}$ & 280 & 16.23 & $40-280$ & 6 & 5.55 & & 0.90 \\
\hline $\begin{array}{l}\text { Cary } \\
\text { USGS Mell GH4 }\end{array}$ & $32^{\circ} 48^{\prime} 21^{*}$ & $90^{\circ} 55^{\prime} 31^{\circ}$ & 190 & 17.11 & $120-190$ & 10 & 4.68 & & 0.80 \\
\hline Matchez & $31^{\circ} 27^{\prime} 52^{\prime}$ & $92^{\circ} 20^{\prime} 22^{\prime \prime}$ & 260 & 27.96 & $220-260$ & 13 & 5.07 & & 1.42 \\
\hline \multicolumn{10}{|l|}{ Lovisians } \\
\hline Bogalusa & $30^{\circ} 49^{\prime} 00^{\prime \prime}$ & $89^{\circ} 54^{\prime} 26^{\prime \prime}$ & 440 & 17.71 & $60-440$ & 13 & 5.90 & & 1.04 \\
\hline $\begin{array}{l}\text { Batan Rouge } \\
\text { (south) }\end{array}$ & $30^{\circ} 25^{\prime} 09^{\prime \prime}$ & $91^{\circ} 08^{\prime} 27^{\circ}$ & 500 & $\begin{array}{l}22.42 \\
23.64\end{array}$ & $\begin{array}{r}100-260 \\
260-500\end{array}$ & 6 & $\begin{array}{l}5.29 \\
4.42\end{array}$ & $\begin{array}{l}1.19 \\
1.06\end{array}$ & $1.10^{8}$ \\
\hline $\begin{array}{l}\text { Baton Rouge } \\
\text { (north) }\end{array}$ & $30^{\circ} 26^{\prime} 14^{\prime \prime}$ & $91^{\circ} 08^{\prime} 30^{\prime \prime}$ & 500 & $\begin{array}{l}25.64 \\
22.06\end{array}$ & $\begin{array}{l}120-300 \\
300-500\end{array}$ & 4 & 5.07 & $\begin{array}{l}1.30 \\
1.12\end{array}$ & $1.21^{\star}$ \\
\hline Scotlandrille & $30^{\circ} 32^{\prime} 51^{\prime}$ & $92^{\circ} 11^{\prime} 50^{\circ}$ & 500 & $\begin{array}{l}23.55 \\
19.57\end{array}$ & $\begin{array}{r}80-280 \\
300-500\end{array}$ & 5 & $\begin{array}{l}5.56 \\
6.10\end{array}$ & $\begin{array}{l}1.30 \\
1.19\end{array}$ & $1.24^{8}$ \\
\hline Bastrop & $32^{\prime} 43^{\prime} 52^{\prime}$ & $91^{\circ} 53^{\prime} 10^{\prime \prime}$ & 258 & $\begin{array}{l}26.54 \\
37.45\end{array}$ & $\begin{array}{r}60-120 \\
120-260\end{array}$ & $\mathbf{3}$ & $\begin{array}{l}5.45 \\
4.92\end{array}$ & $\begin{array}{l}1.44 \\
1.64\end{array}$ & $1.72^{2}$ \\
\hline $\begin{array}{l}\text { Bosco } \\
\text { USGS Well ou } 402 \\
\text { Malle } \\
\text { USGS Well un } 79\end{array}$ & $\begin{array}{l}32^{\circ} 17^{\prime} 14^{\prime \prime} \\
32^{\circ} 49^{\prime} 55^{\prime \prime}\end{array}$ & $\begin{array}{l}92^{\circ} 04^{\prime} 14^{\prime} \\
22^{\circ} 08^{\prime} 40^{\prime}\end{array}$ & $\begin{array}{l}236 \\
236\end{array}$ & $\begin{array}{l}20.79 \\
29.00 \\
22.58 \\
34.98\end{array}$ & $\begin{array}{r}10-100 \\
120-236 \\
60-100 \\
100-236\end{array}$ & 23 & $\begin{array}{l}4.07 \\
6.46\end{array}$ & $\begin{array}{l}0.85 \\
1.18 \\
1.46 \\
2.26\end{array}$ & $\begin{array}{l}1.078 \\
2.08\end{array}$ \\
\hline $\begin{array}{l}\text { Devillie } \\
\text { usGs Mell \& } 893\end{array}$ & $31^{\circ} 23^{\circ} 39^{*}$ & $92^{\circ} 09^{\prime} 45^{\prime \prime}$ & 250 & 24.94 & $40-250$ & 8 & 4.51 & & 1.12 \\
\hline $\begin{array}{l}\text { Kelly } \\
\text { uscs vell Ca 86b }\end{array}$ & $32^{\circ} 01^{\prime} 54^{\prime \prime}$ & $92^{\circ} 16^{\circ} 46^{\prime \prime}$ & 172 & $\begin{array}{l}26.56 \\
44.23\end{array}$ & $\begin{array}{r}60-120 \\
120-172\end{array}$ & 7 & 6.72 & $\begin{array}{l}1.52 \\
2.53\end{array}$ & $1.99 \approx$ \\
\hline $\begin{array}{l}\text { Calhoun } \\
\text { uscs tell ou } 443\end{array}$ & $32^{\circ} 31^{\prime} 00^{\prime \prime}$ & $92^{\circ} 16^{\circ} 58^{\prime \prime}$ & 260 & 31.08 & $60-260$ & 10 & 5.12 & & 1.59 \\
\hline $\begin{array}{l}\text { Truxno } \\
\text { usc5 vell Un } 80\end{array}$ & $32^{\circ} 49^{\prime} 17^{\prime \prime}$ & $22^{\circ} 24^{\prime} 20^{\prime \prime}$ & 215 & 33.13 & $60-215$ & 9 & 6.49 & & 2.13 \\
\hline $\begin{array}{l}\text { S1kes } \\
\text { USCS Mell W } 172\end{array}$ & $32^{\prime 05}$ '4l" & $92^{\circ} 29^{\prime} 16^{\prime \prime}$ & 200 & 30.30 & $60-200$ & 9 & 5.93 & & 2.80 \\
\hline Yernon & $32^{\circ} 23^{\prime} 57^{\prime}$ & $92^{\circ} 34^{\prime} 17^{\prime}$ & 217 & $\begin{array}{l}18.76 \\
29.11\end{array}$ & $\begin{array}{r}40-120 \\
120-220\end{array}$ & 3 & $\begin{array}{l}5.45 \\
4.92\end{array}$ & $\begin{array}{l}1.04 \\
1.43\end{array}$ & $1.26^{8}$ \\
\hline $\begin{array}{l}\text { North Modge } \\
\text { USGS Mell Ja } 156\end{array}$ & $32^{\circ} 17^{\prime} 30^{\circ}$ & $22^{\circ} 37^{\prime} 37^{\circ}$ & 200 & 23.74 & $40-200$ & 7 & 5.40 & & 1.28 \\
\hline $\begin{array}{l}\text { Minnfield } \\
\text { uscs tell } \text { * } 177\end{array}$ & $31^{\circ} 53^{\prime} 38^{\prime}$ & $22^{\circ} 40^{\circ} 00^{\prime \prime}$ & 2215 & $\begin{array}{l}13.20 \\
27.65\end{array}$ & $\begin{array}{r}20-80 \\
100-215\end{array}$ & 5 & 5.73 & $\begin{array}{l}0.76 \\
1.58\end{array}$ & $1.30^{2}$ \\
\hline $\begin{array}{l}\text { Bernice } \\
\text { uSGS Mell CI } 151\end{array}$ & $32^{\circ} 00^{\prime} 08^{\circ}$ & $92^{*} 41^{\prime} 35^{\prime \prime}$ & 220 & $\begin{array}{l}22.73 \\
32.17\end{array}$ & $\begin{array}{r}60-120 \\
120-220\end{array}$ & 8 & 6.17 & $\begin{array}{l}1.40 \\
1.98\end{array}$ & $1.76^{2}$ \\
\hline $\begin{array}{l}\text { Junetion city } \\
\text { usGS Nell Cl } 149\end{array}$ & $33^{\circ} 00^{\prime} 02^{\prime \prime}$ & $92^{\circ} 44^{\prime} 59^{\circ}$ & 230 & 24.98 & $120-230$ & 9 & 6.02 & & 1.50 \\
\hline $\begin{array}{l}\text { Marsarts } \\
\text { uSGS Mell Cl } 136\end{array}$ & $36^{\circ} 39^{\prime} 43^{\prime \prime}$ & $92^{\circ} 57^{\prime} 36^{*}$ & 258 & 27.48 & $60-258$ & 6 & 6.50 & & 1.79 \\
\hline Simpson & $31^{\circ} 15^{\prime} 44^{\prime \prime}$ & $93^{\circ} 00^{\prime} 25^{\circ}$ & 378 & 26.82 & $60-380$ & 7 & 4.78 & & 1.28 \\
\hline
\end{tabular}

-velghted average. 


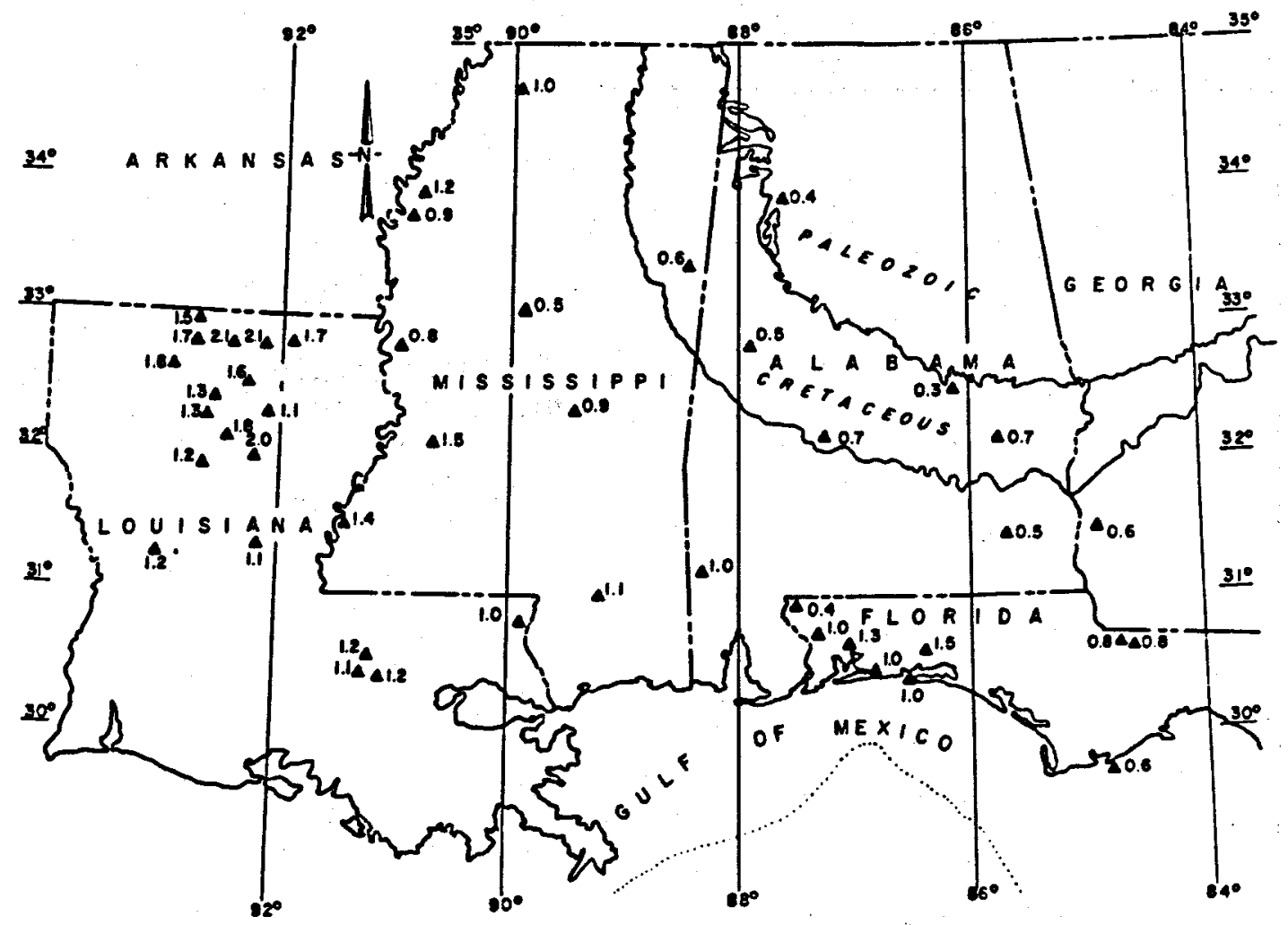

Fig. 3.

Heat-flow values (in heat-flow units) for the Gulf Coastal Plain (from Smith, Dees, and Harrelson, 1981).

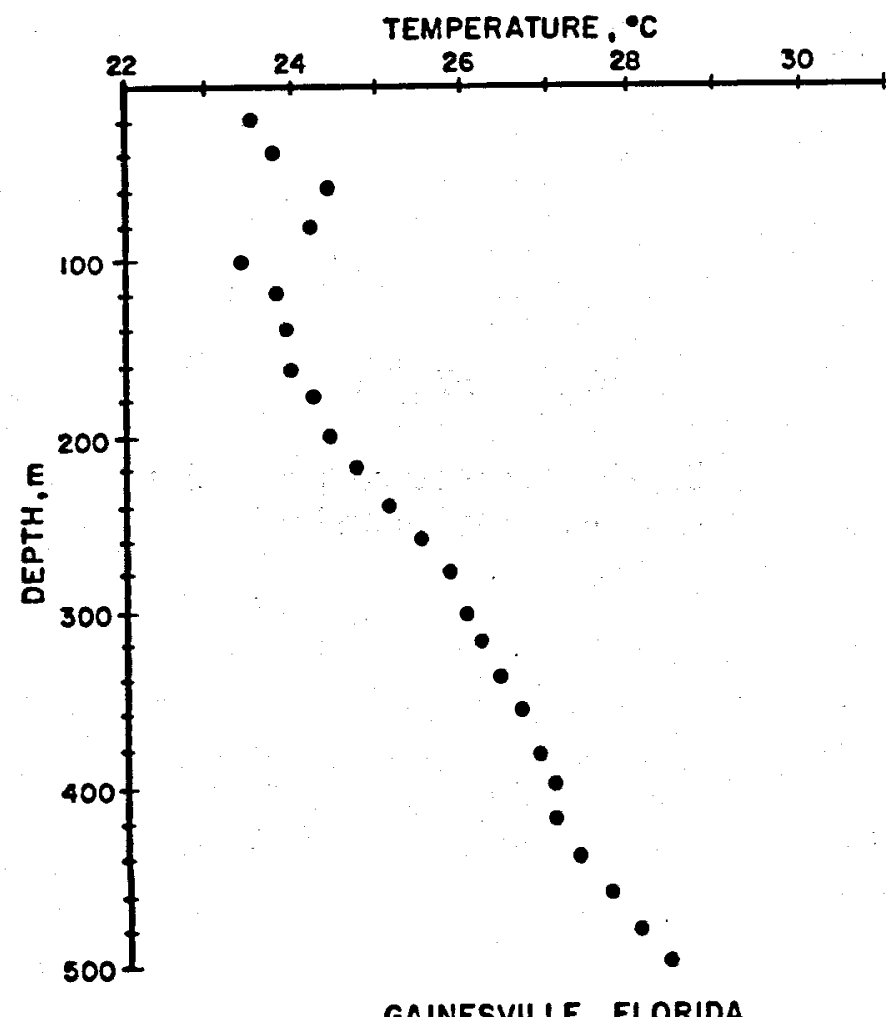

Fig. 4 .

Depth/temperature profile in Gainesville, Florida well.

GAINESVILLE, FLORIDA 


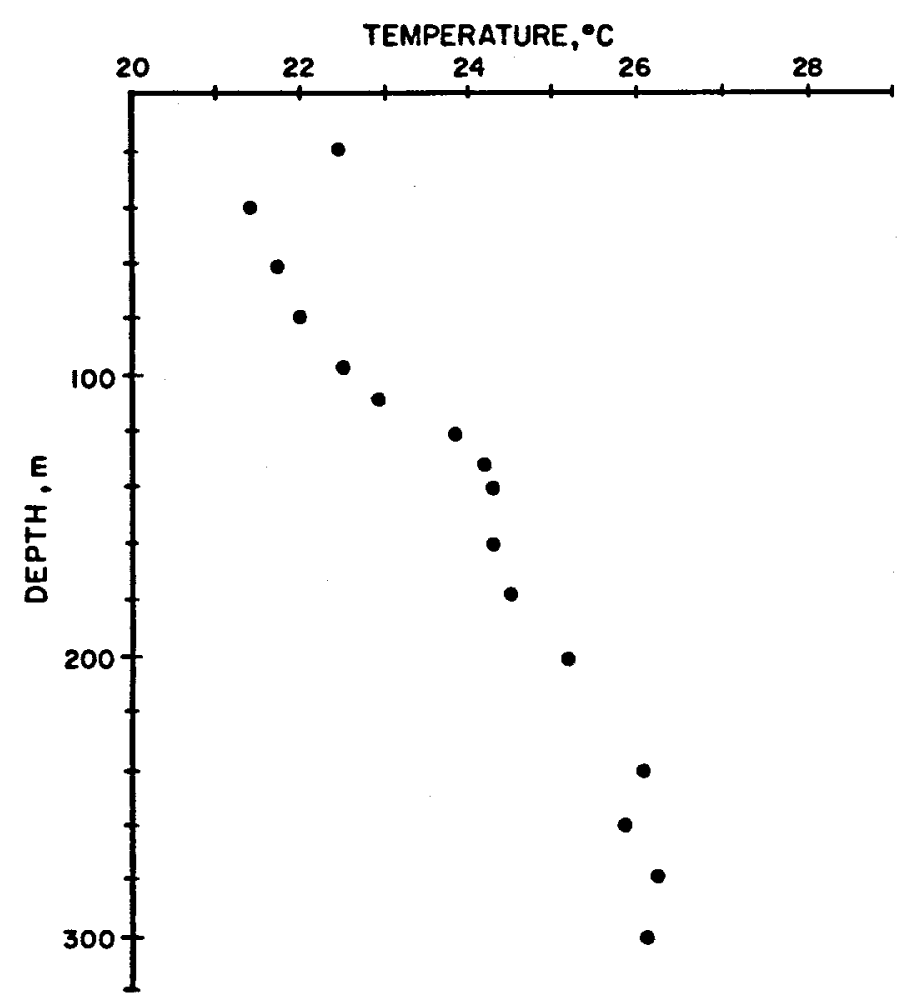

Fig. 5 .

Depth/temperature profile in Wellborn, Florida well.

WELLBORN, FLORIDA

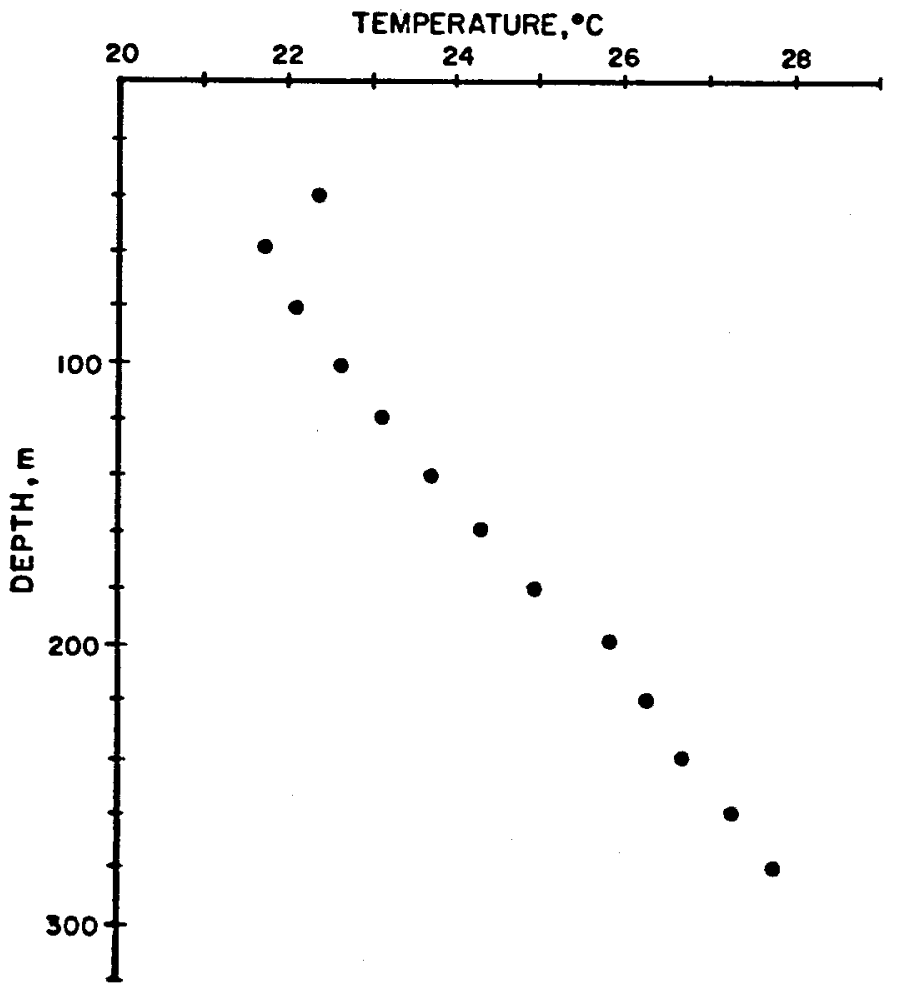

Fig. 6 .

Depth/temperature profile in Gretna, Florida well. 


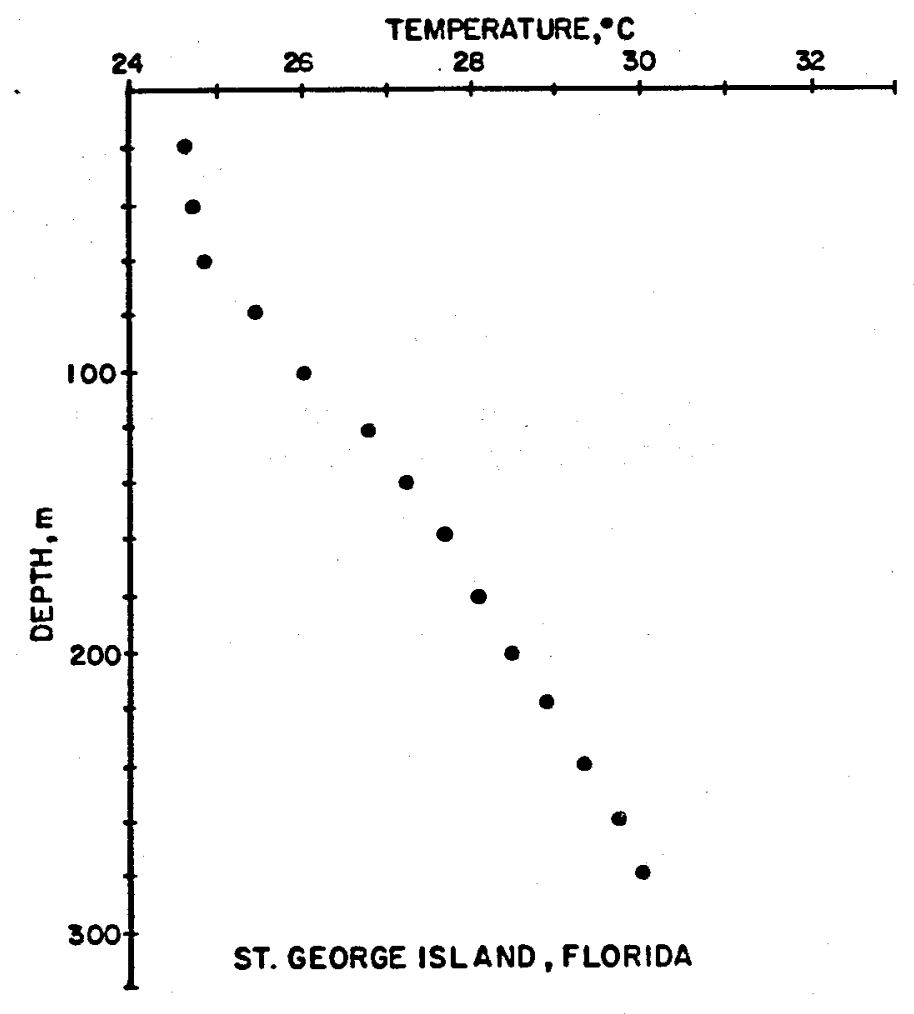

Fig. 7.

Depth/temperature profile in St. George Island, Florida well.

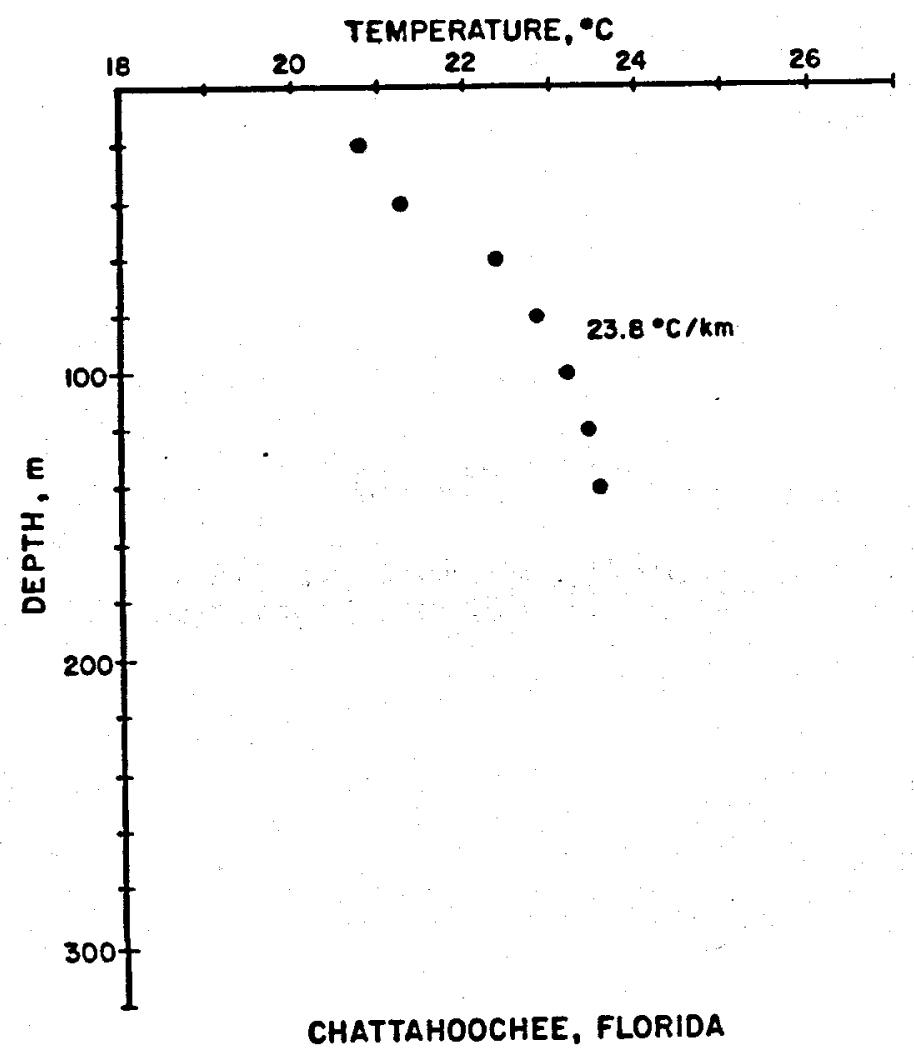

Fig. 8.

Depth/temperature profile in Chattahoochee, Florida well. 


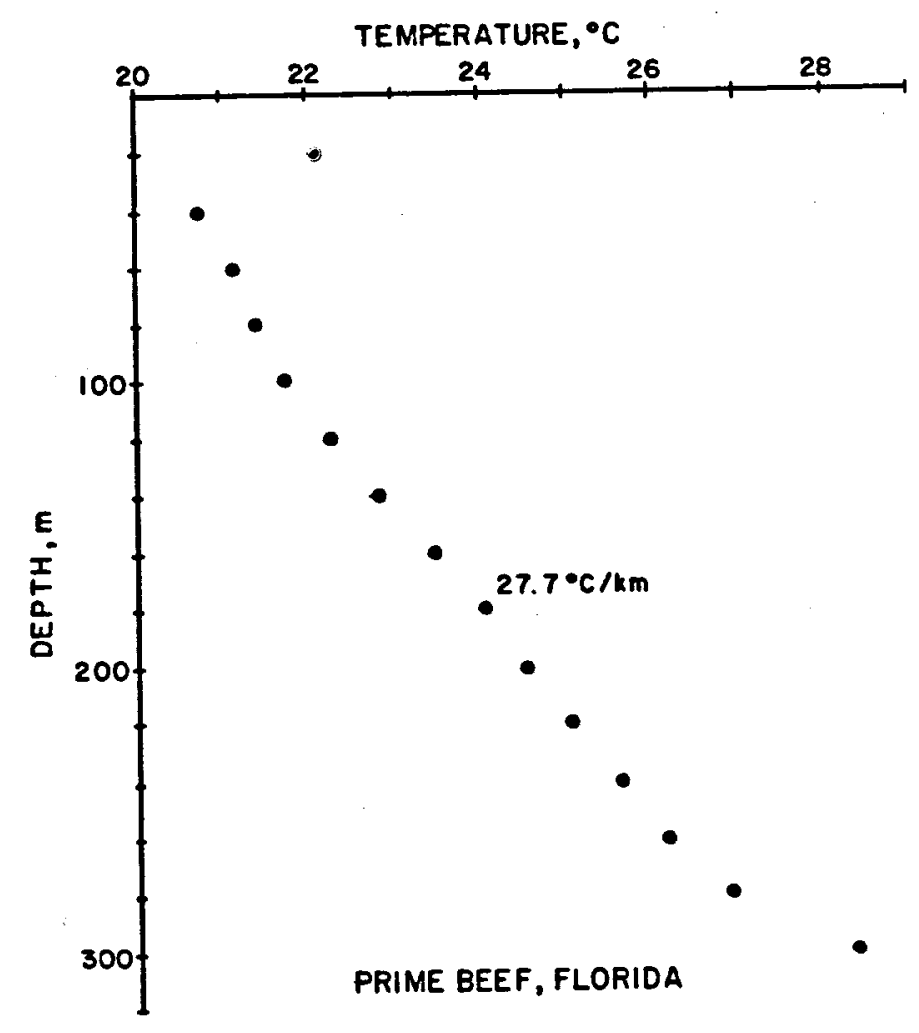

Fig. 9.

Depth/temperature profile in Prime Beef, Florida well.

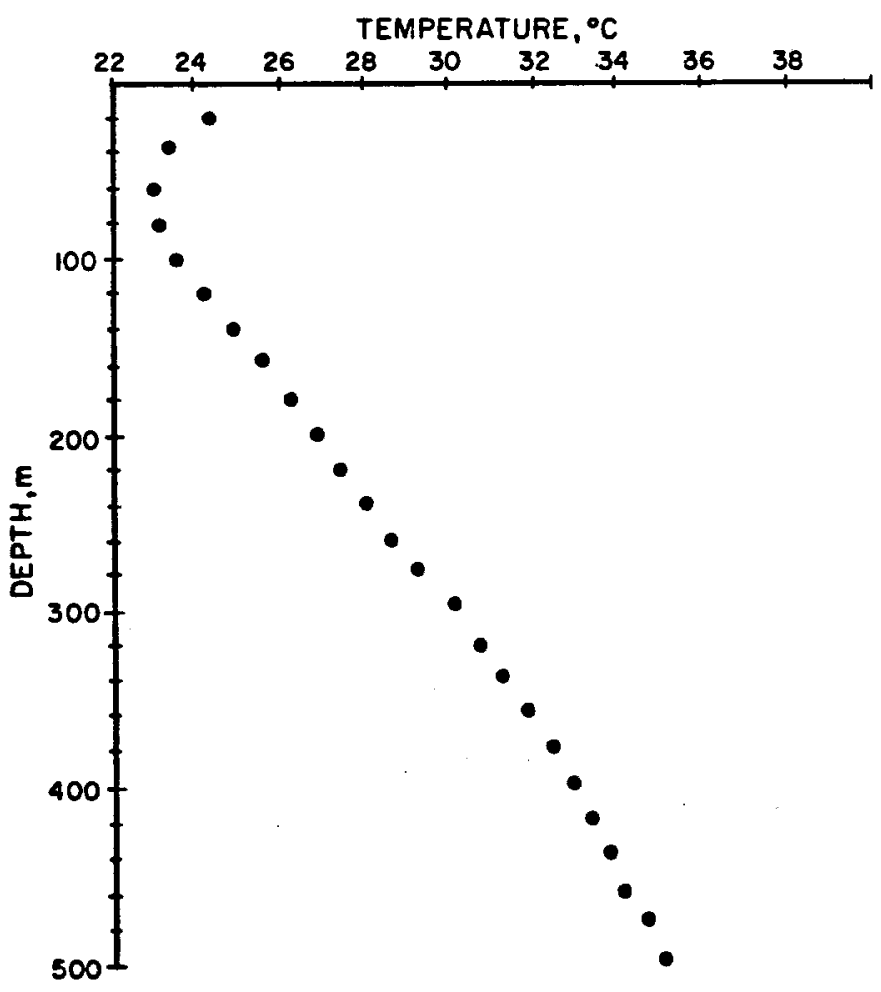

Fig. 10.

Depth/temperature profile in Ft. Walton Beach, Florida well.

FT. WALTON BEACH, FLORIDA 


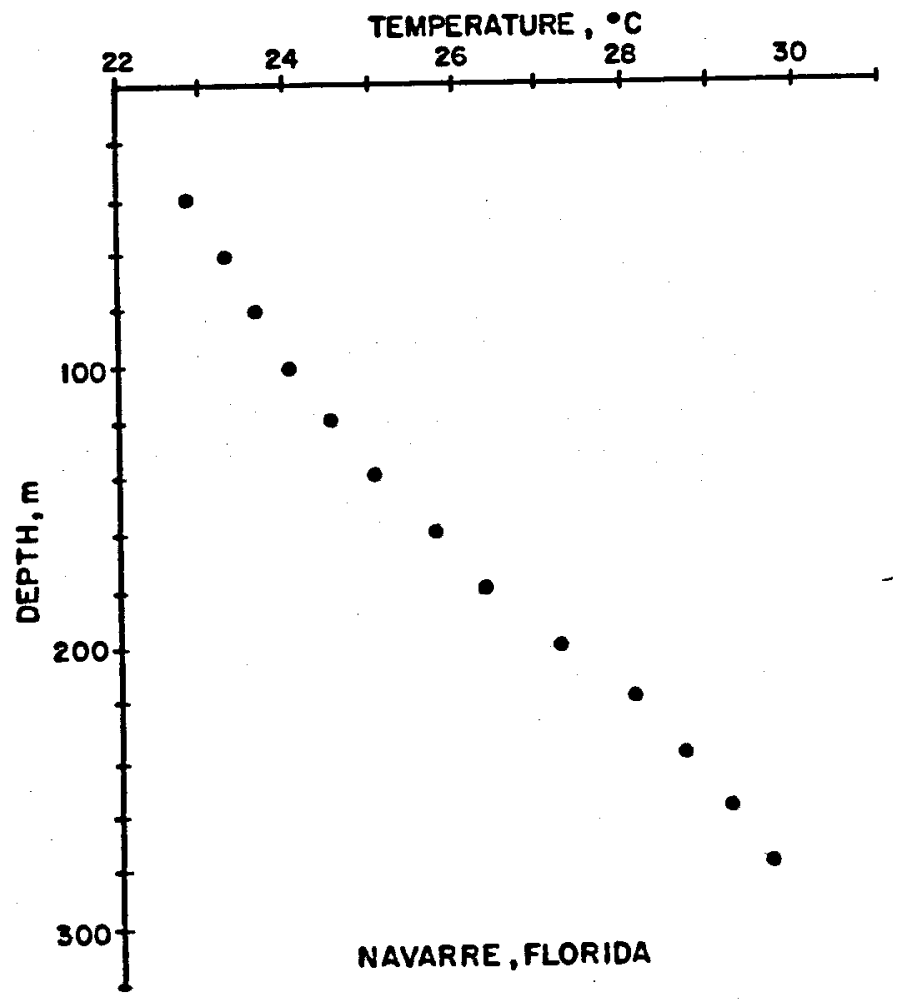

Fig. 11.

Depth/temperature profile in Navarre, Florida well.

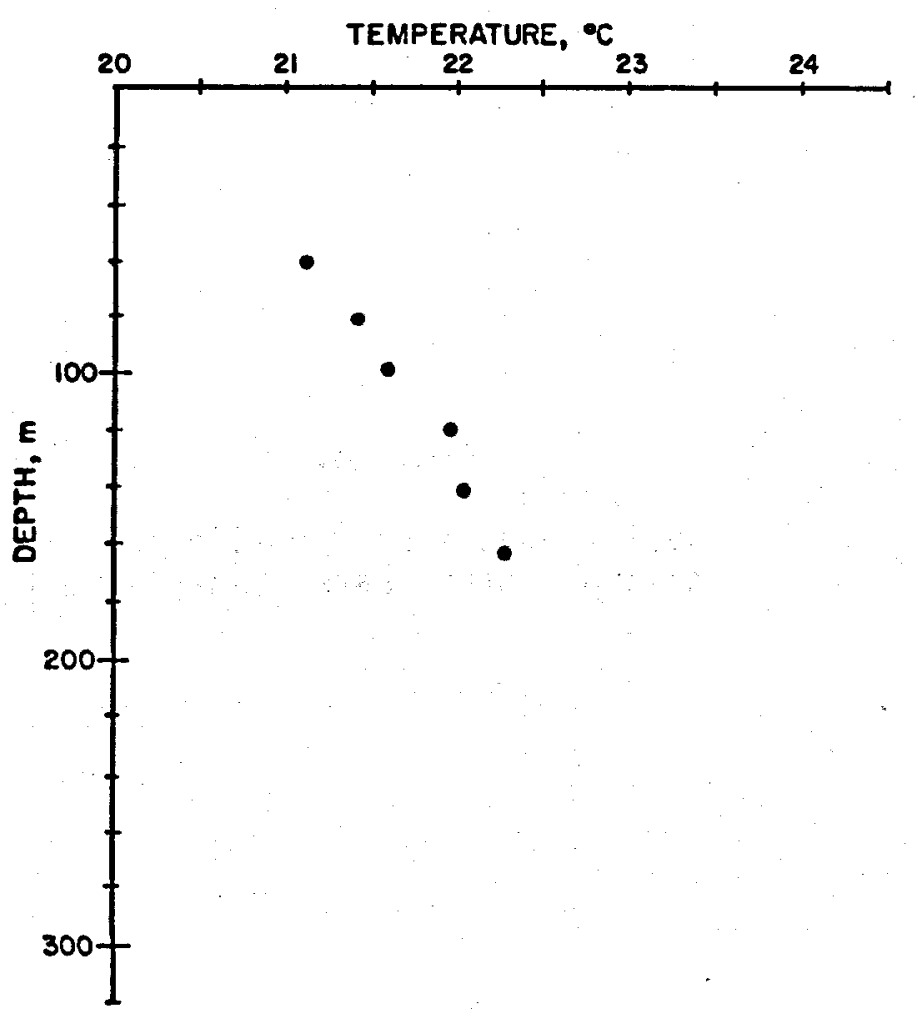

Fig. 12 .

Depth/temperature profile in Camp Henderson, Florida well.

CAMP HENDERSON, FLORIDA 


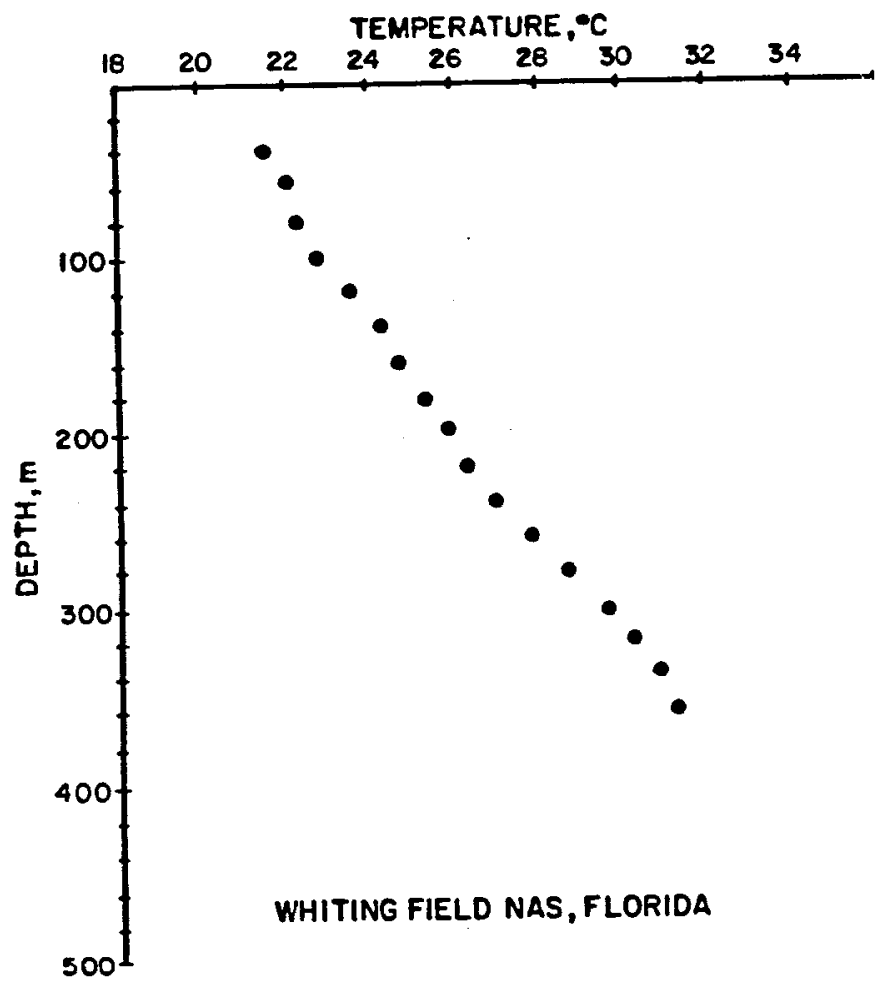

Fig. 13.

Depth/temperature profile in Whiting Field NAS, Florida well.

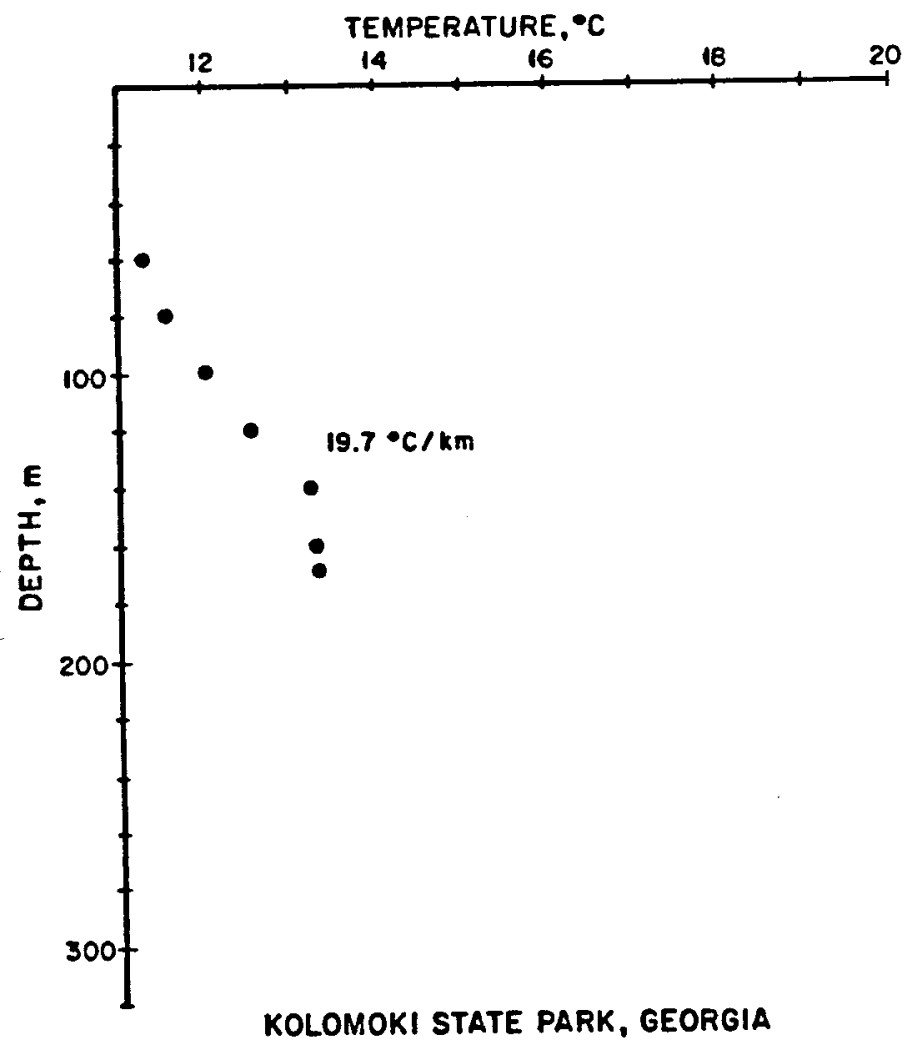

Fig. 14 .

Depth/temperature profile in Kolomoki State Park, Georgia well. 


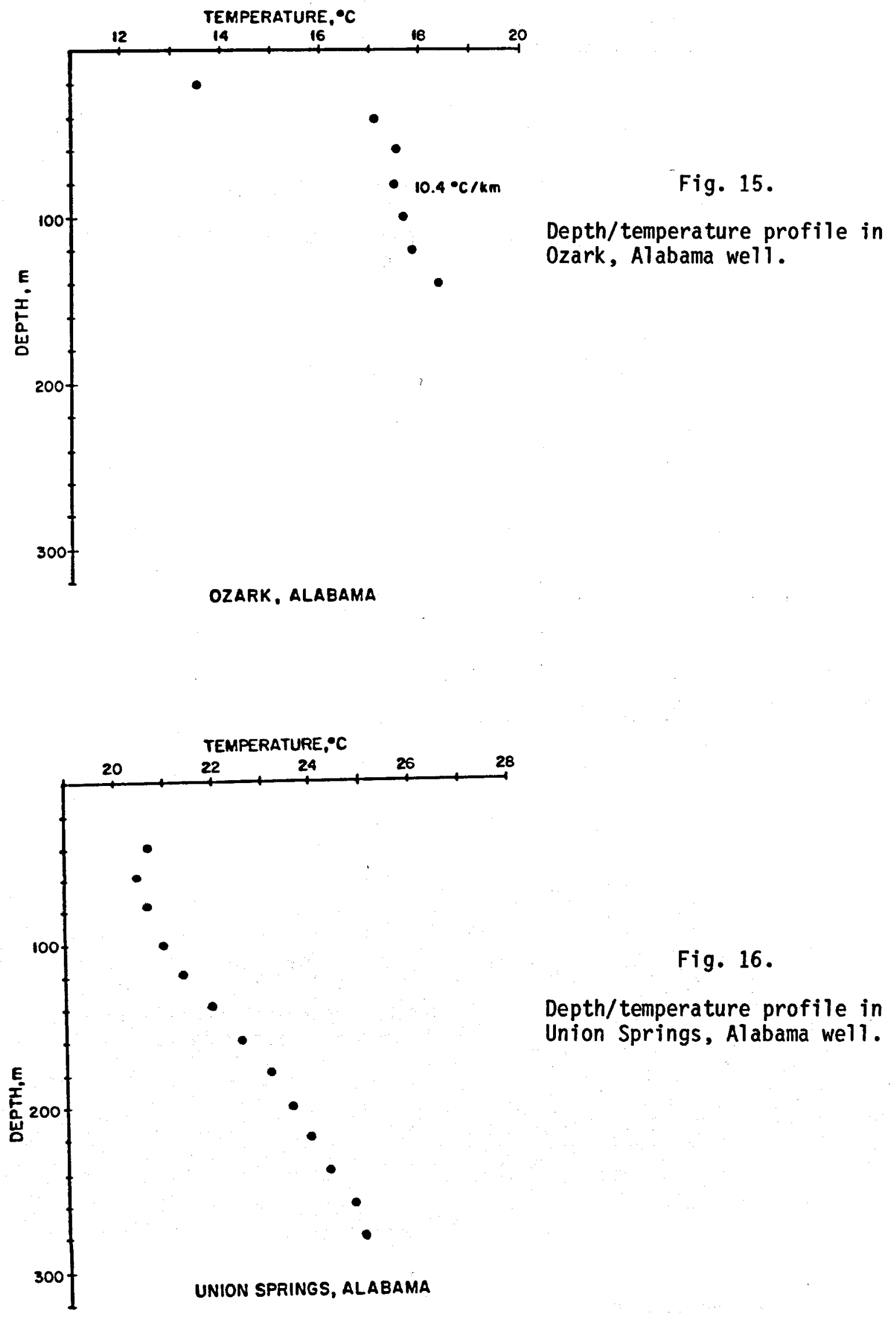




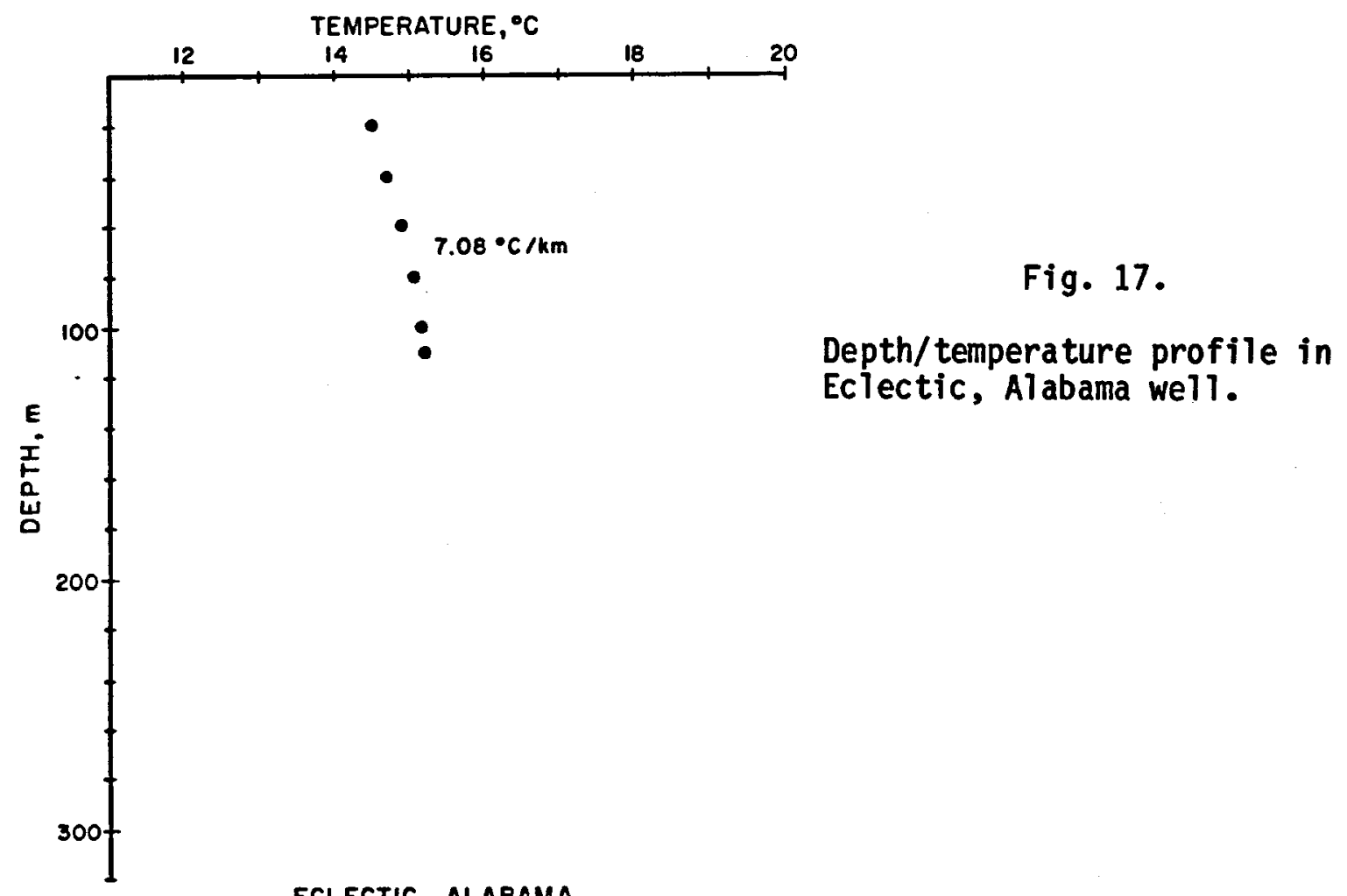

ECLECTIC, ALABAMA

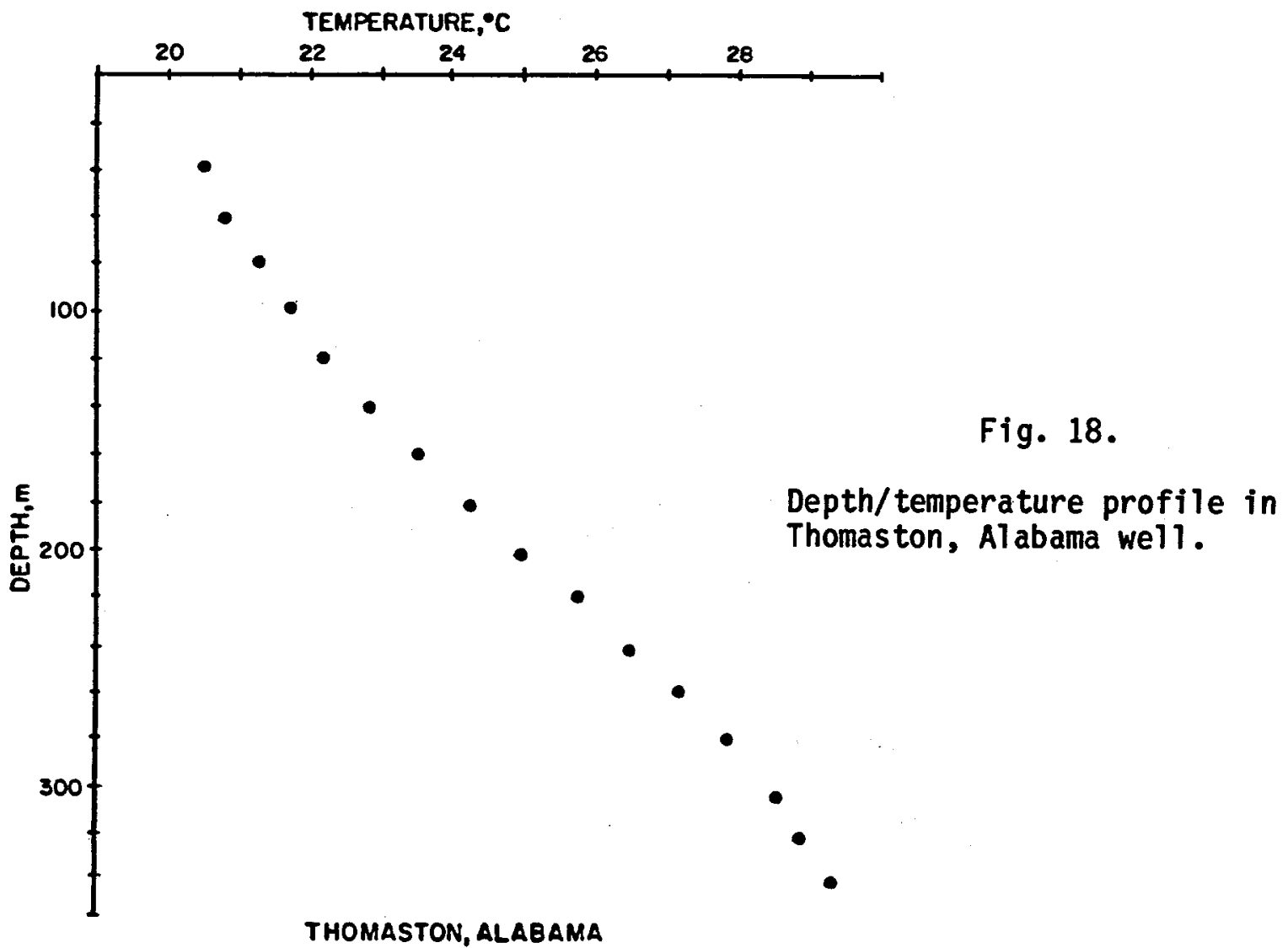




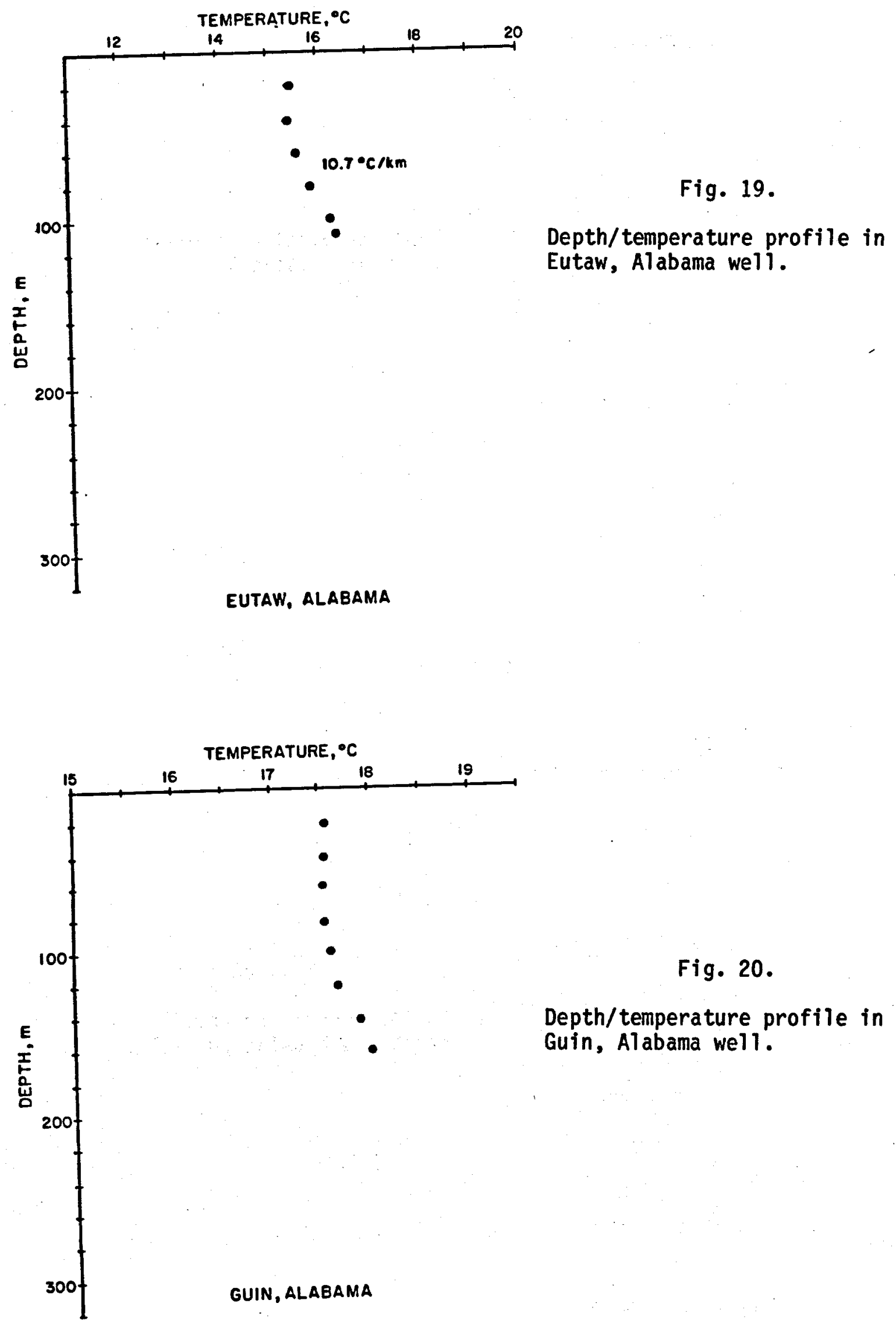



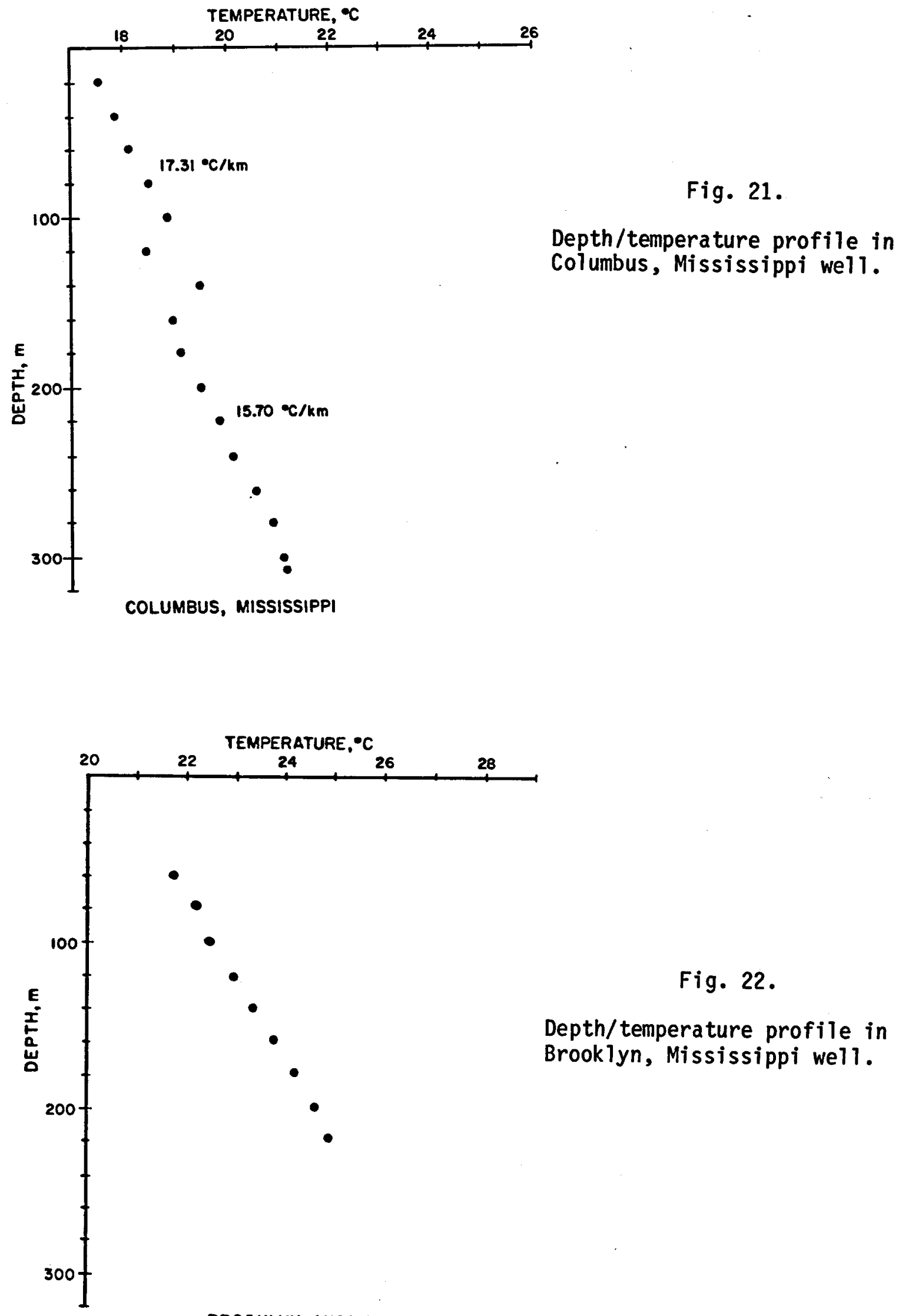

BROOKLYN, MISSISSIPPI 


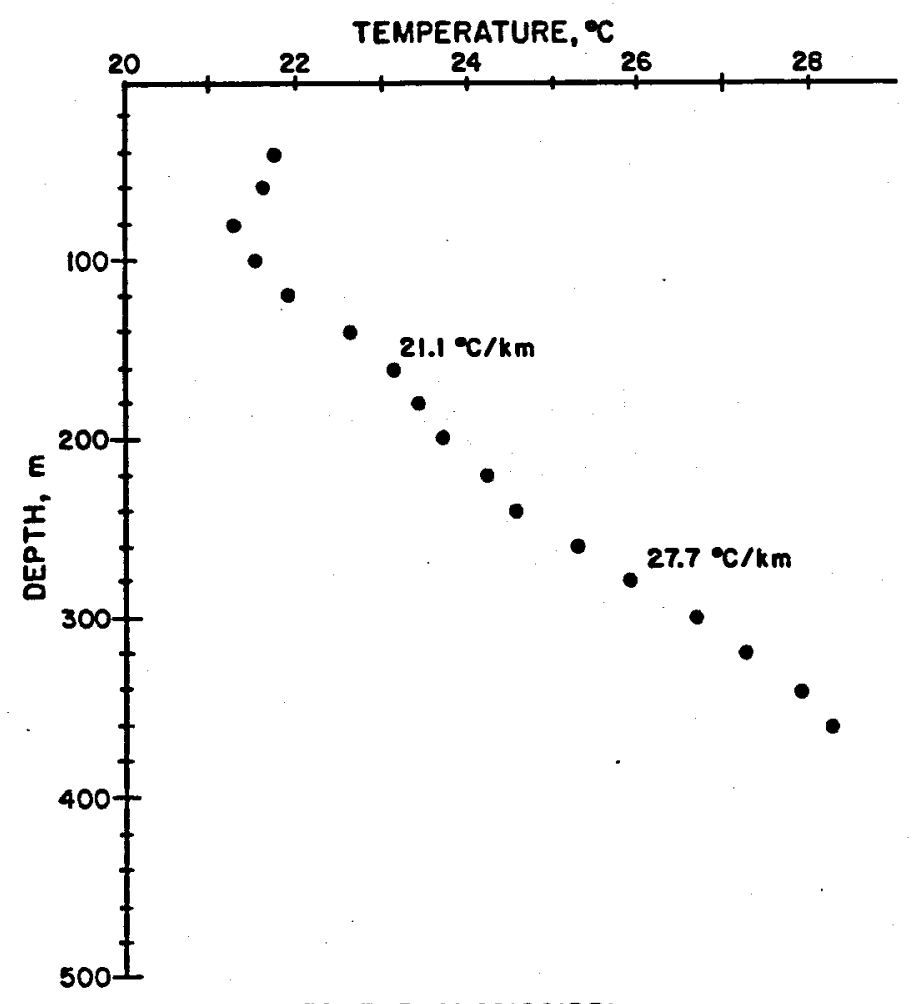

Fig. 23.

Depth/temperature profile in Forest, Mississippi well.

FOREST, MISSISSIPPI

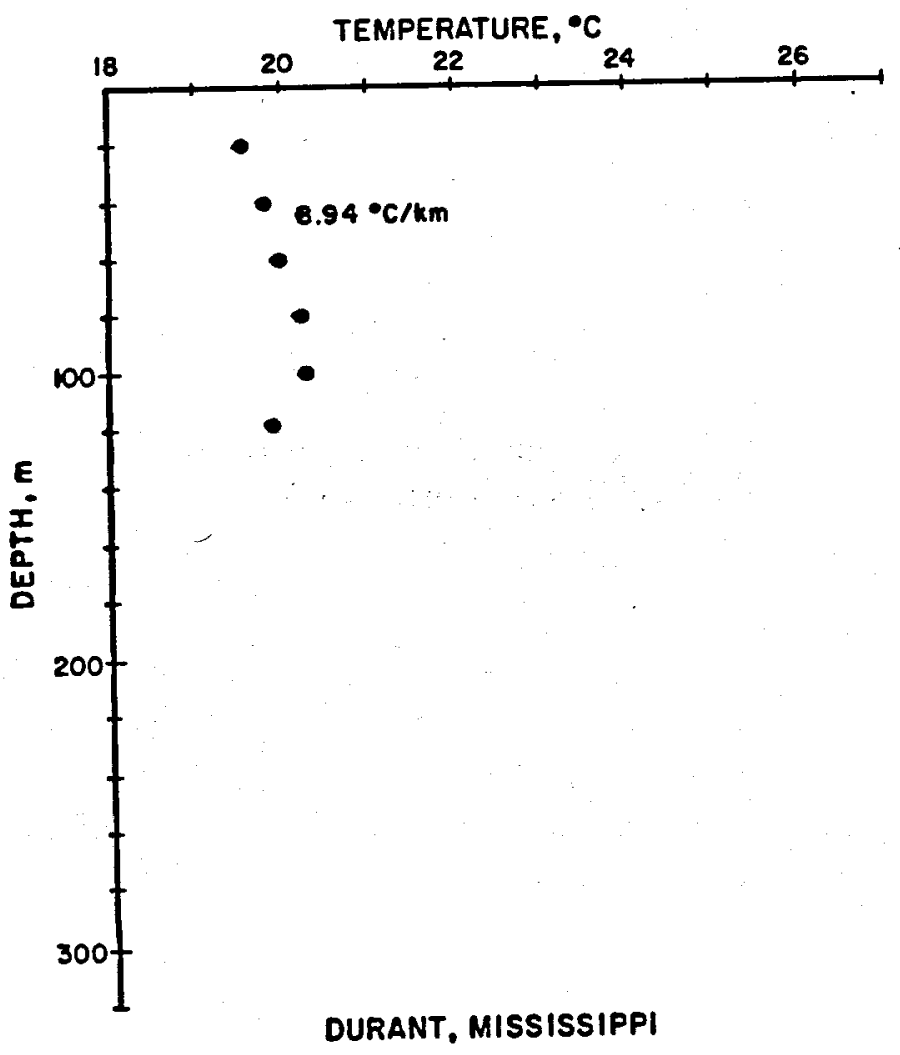

Fig. 24.

Depth/temperature profile in Durant, Mississippi well. 

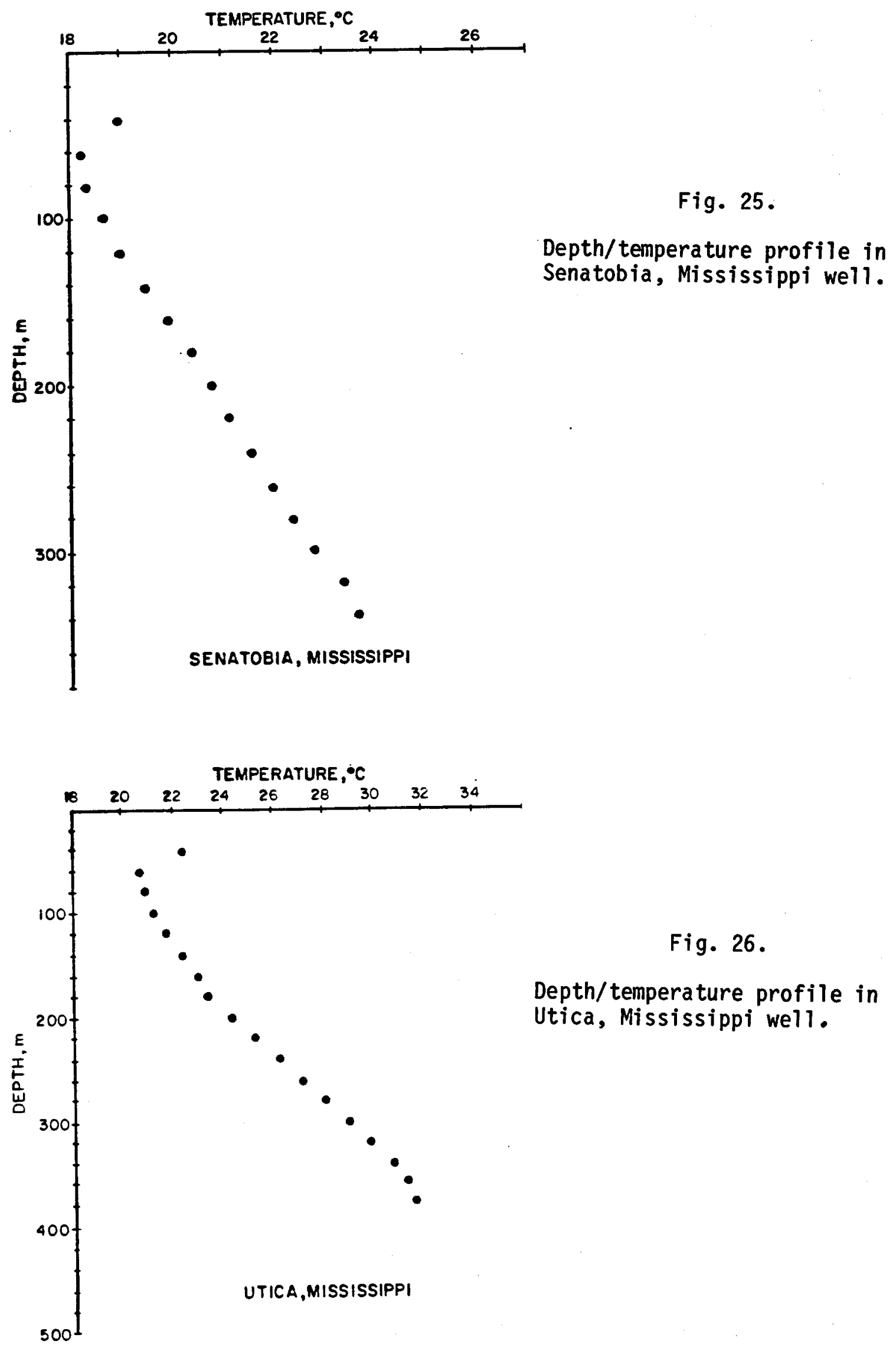

Fig. 26.

Depth/temperature profile in Utica, Mississippi well. 


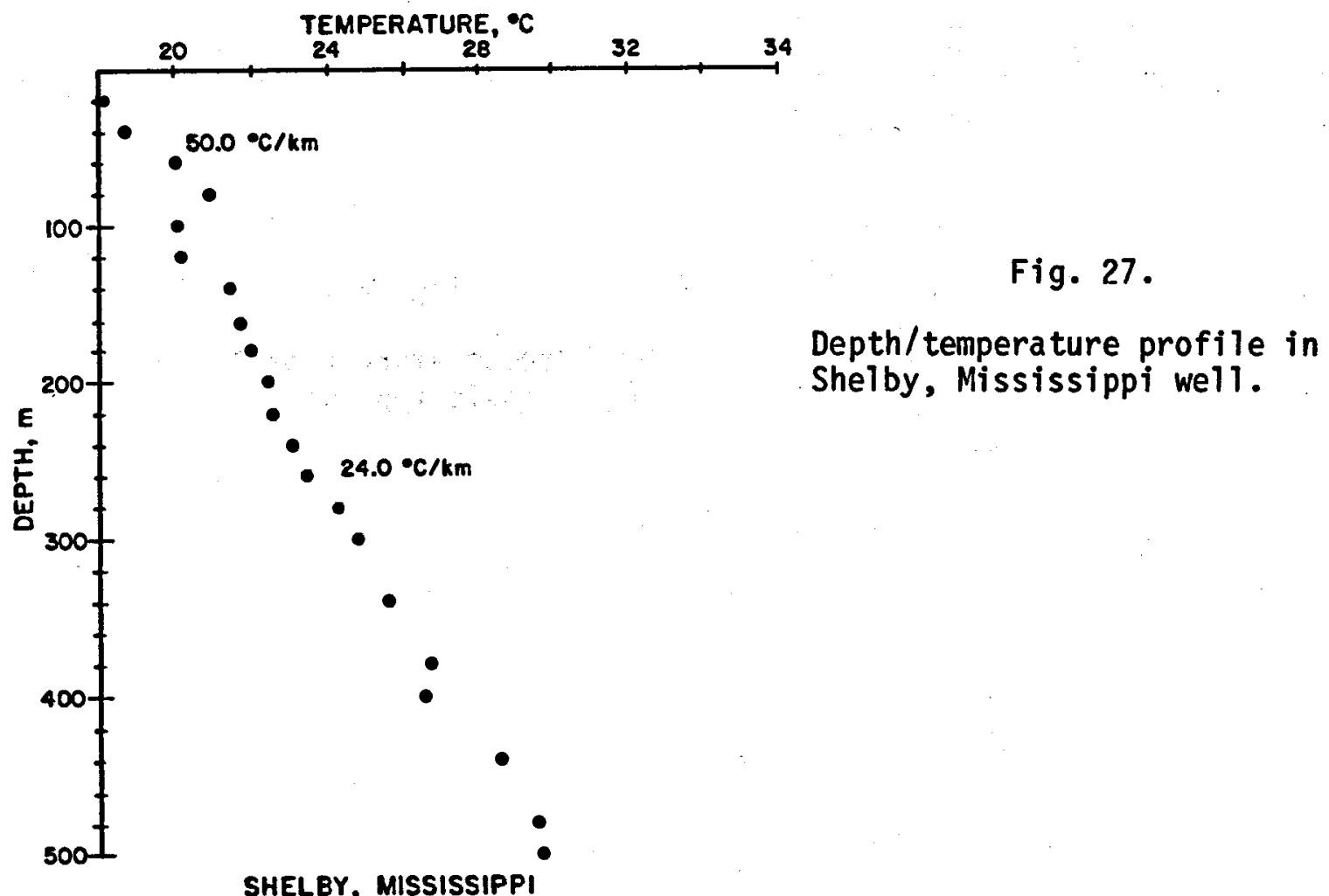

SHELEY, MISSISSIPPI

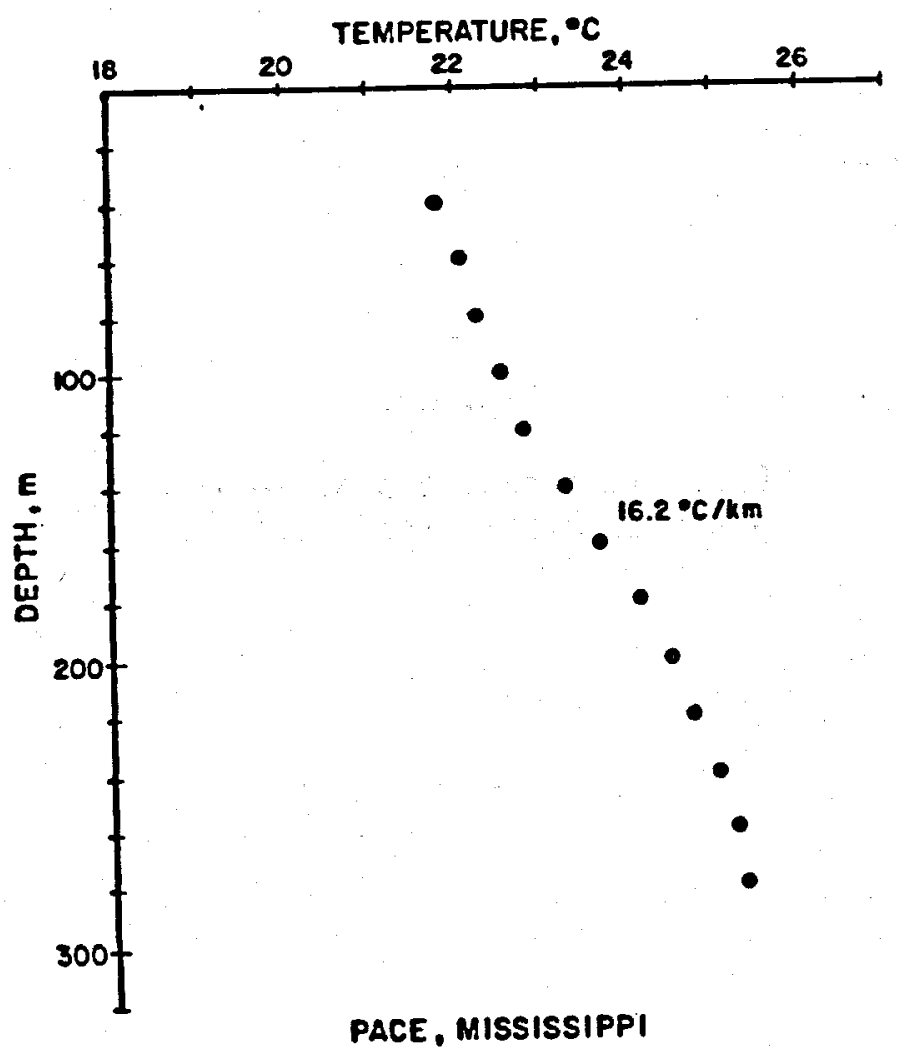

Fig. 28.

Depth/temperature profile in Pace, Mississippi well.

PACE, MISSISSIPPI 


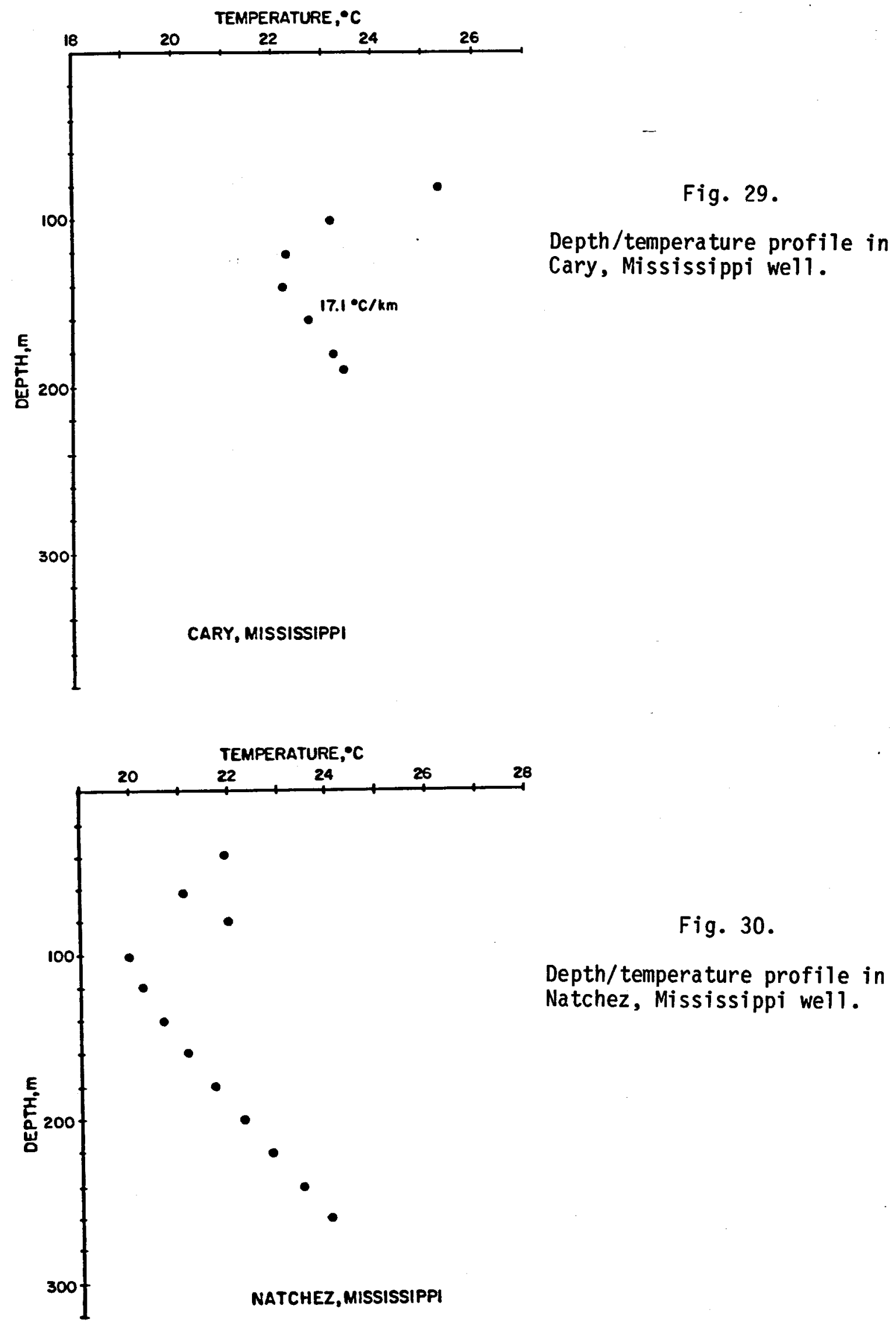




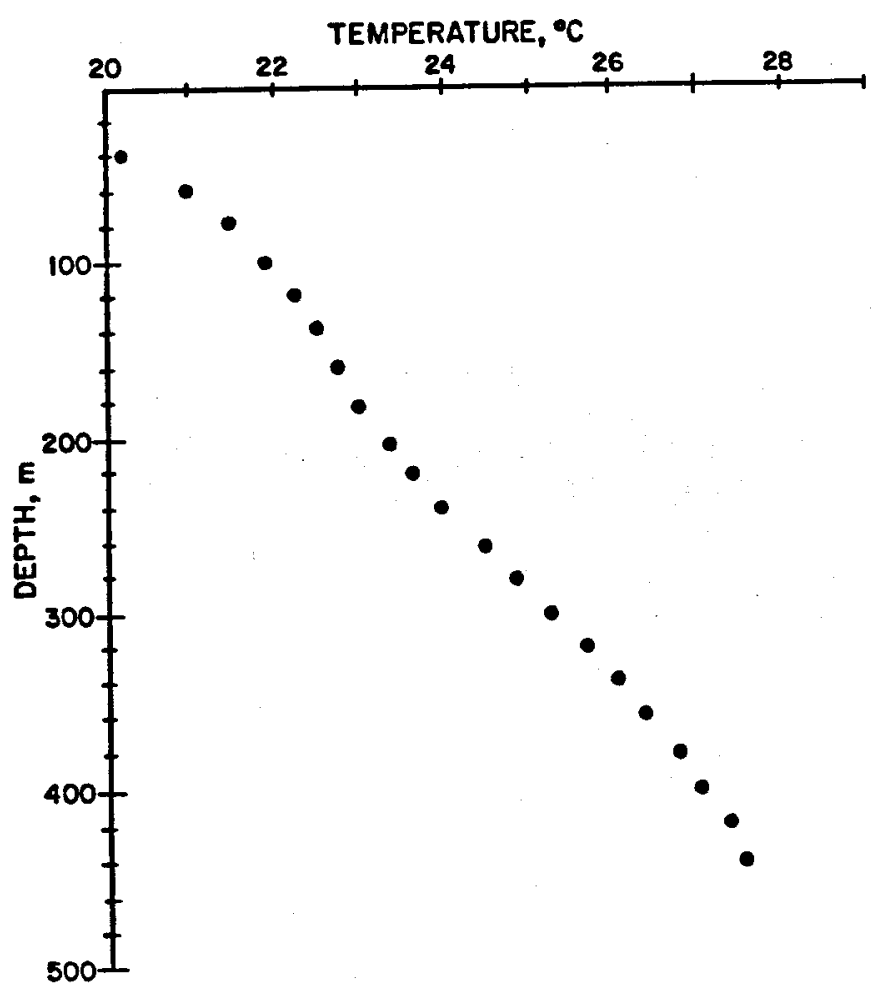

BOGALUSA, LOUISIANA

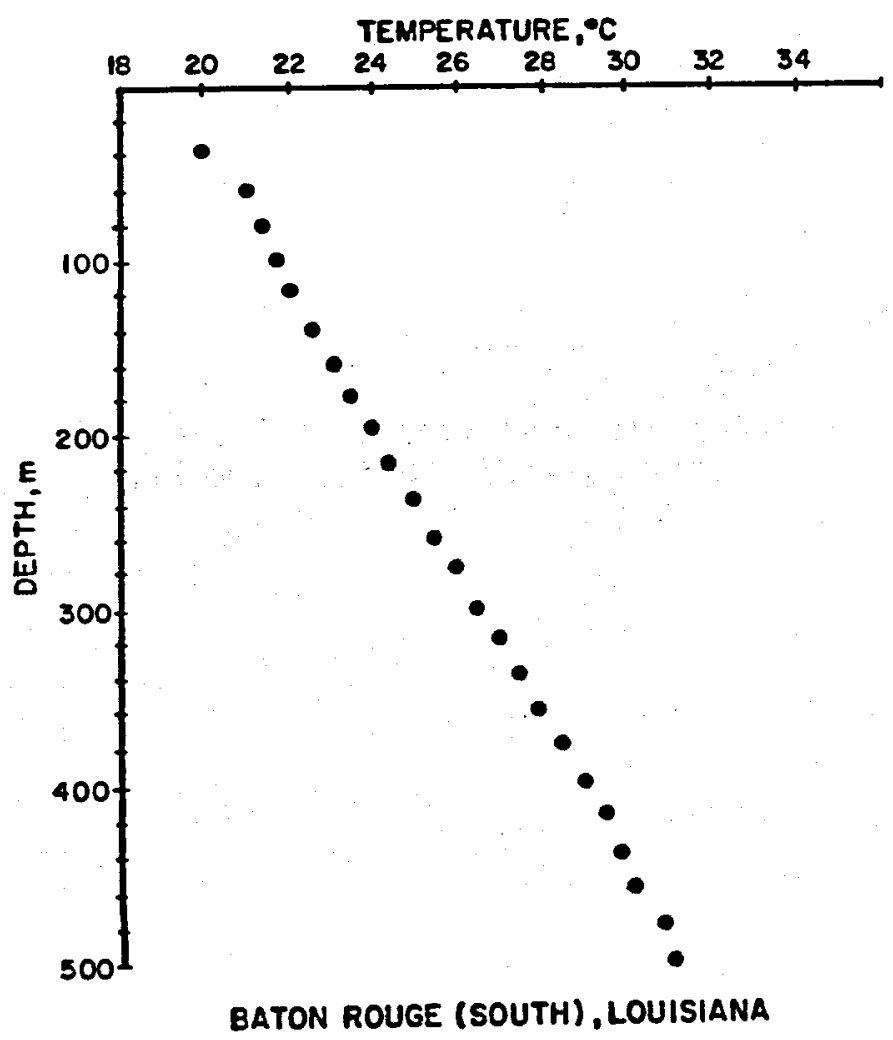

Fig. 31 .

Depth/temperature profile in Bogalusa, Louisiana well.
Fig. 32.

Depth/temperature profile in Baton Rouge (South), Louisiana wel1. 


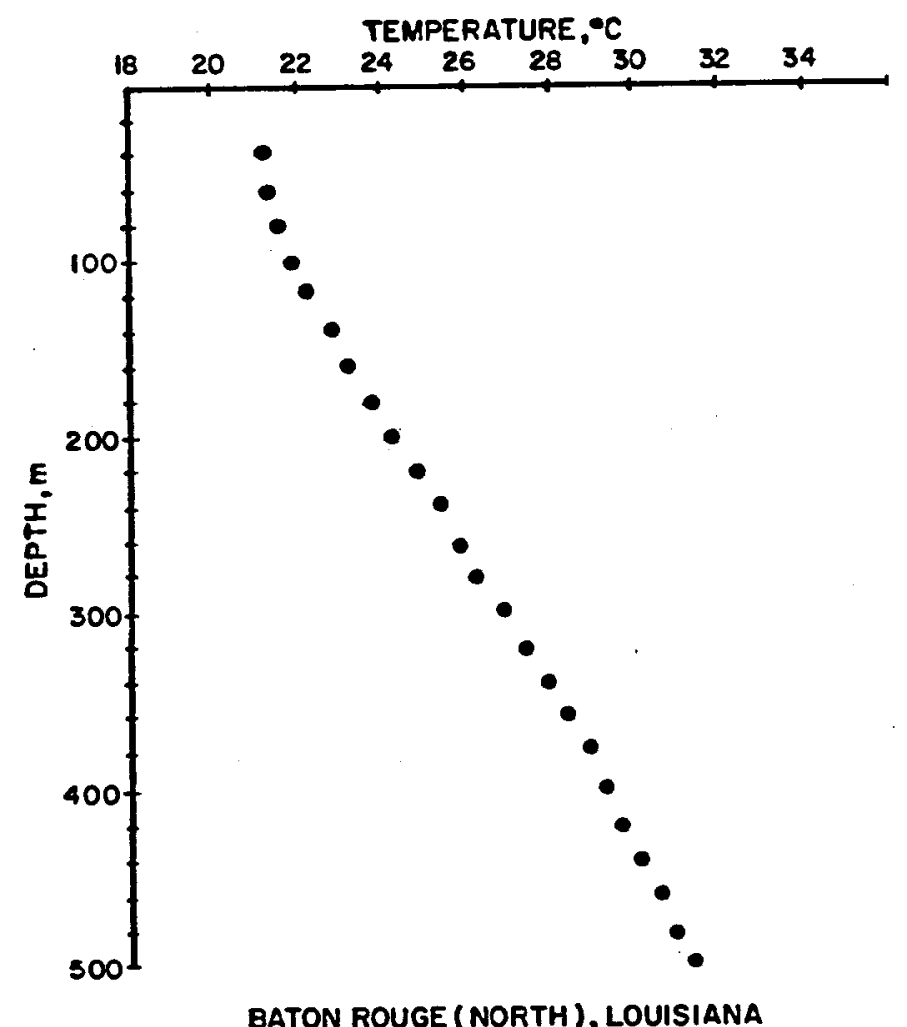

Fig. 33.

Depth/temperature profile in Baton Rouge (North), Louisiana well.

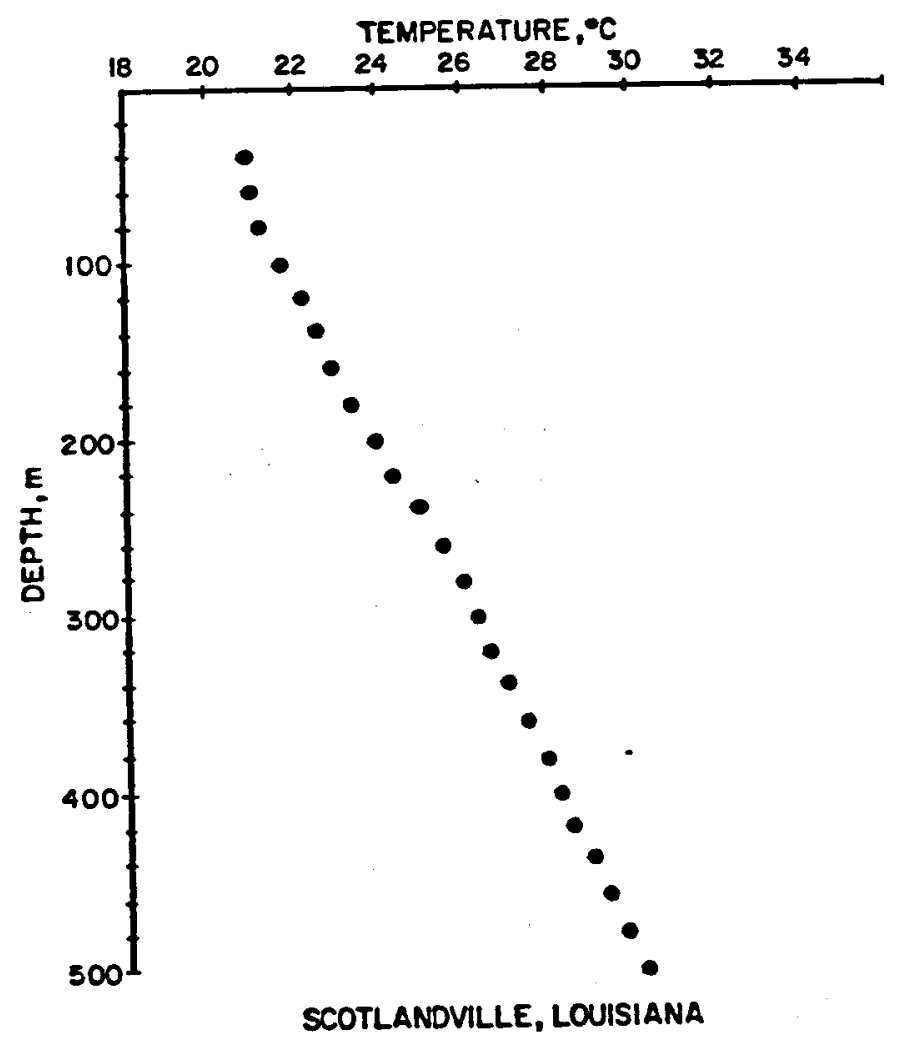

Fig. 34 .

Depth/temperature profile in Scotlandville, Louisiana well. 

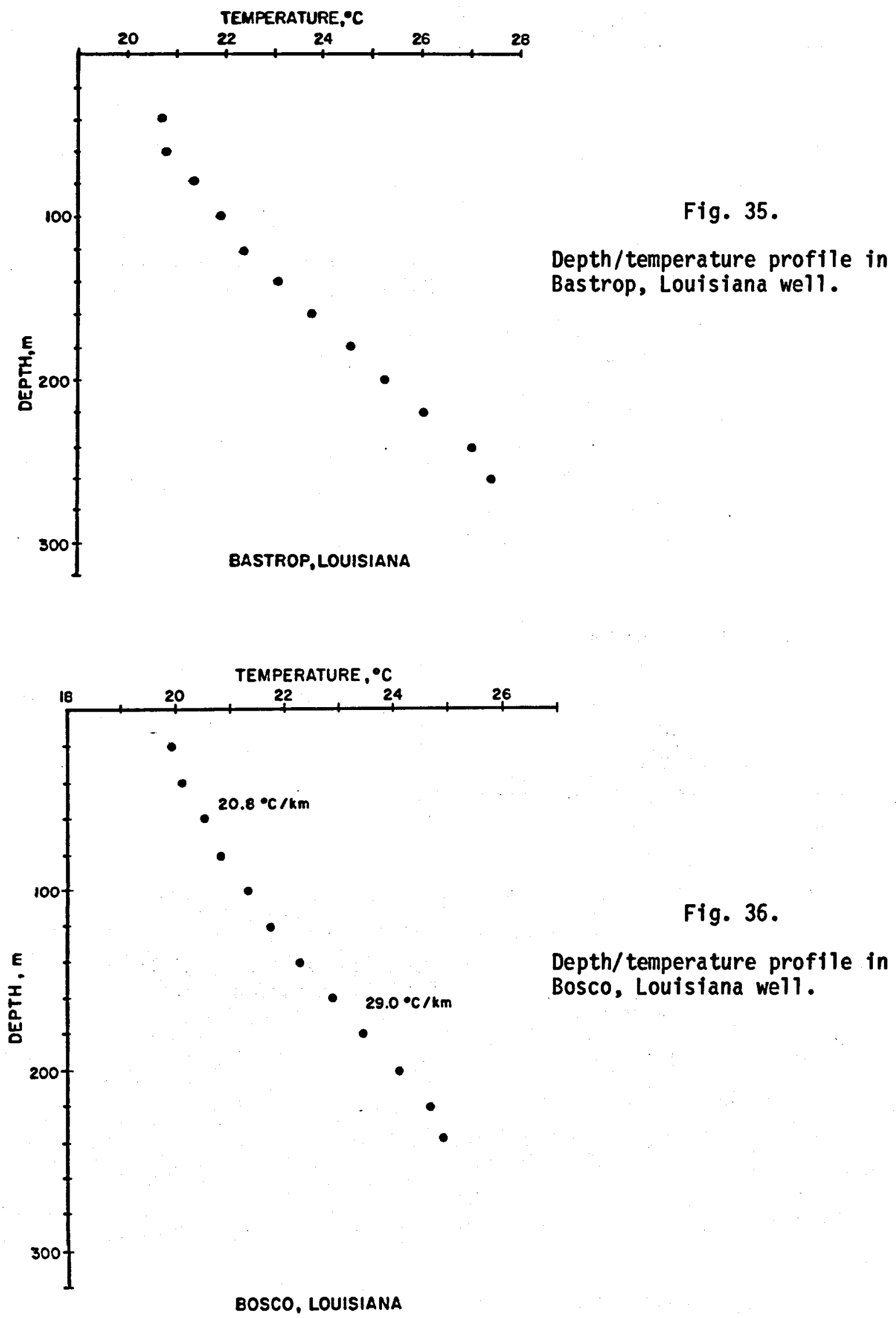

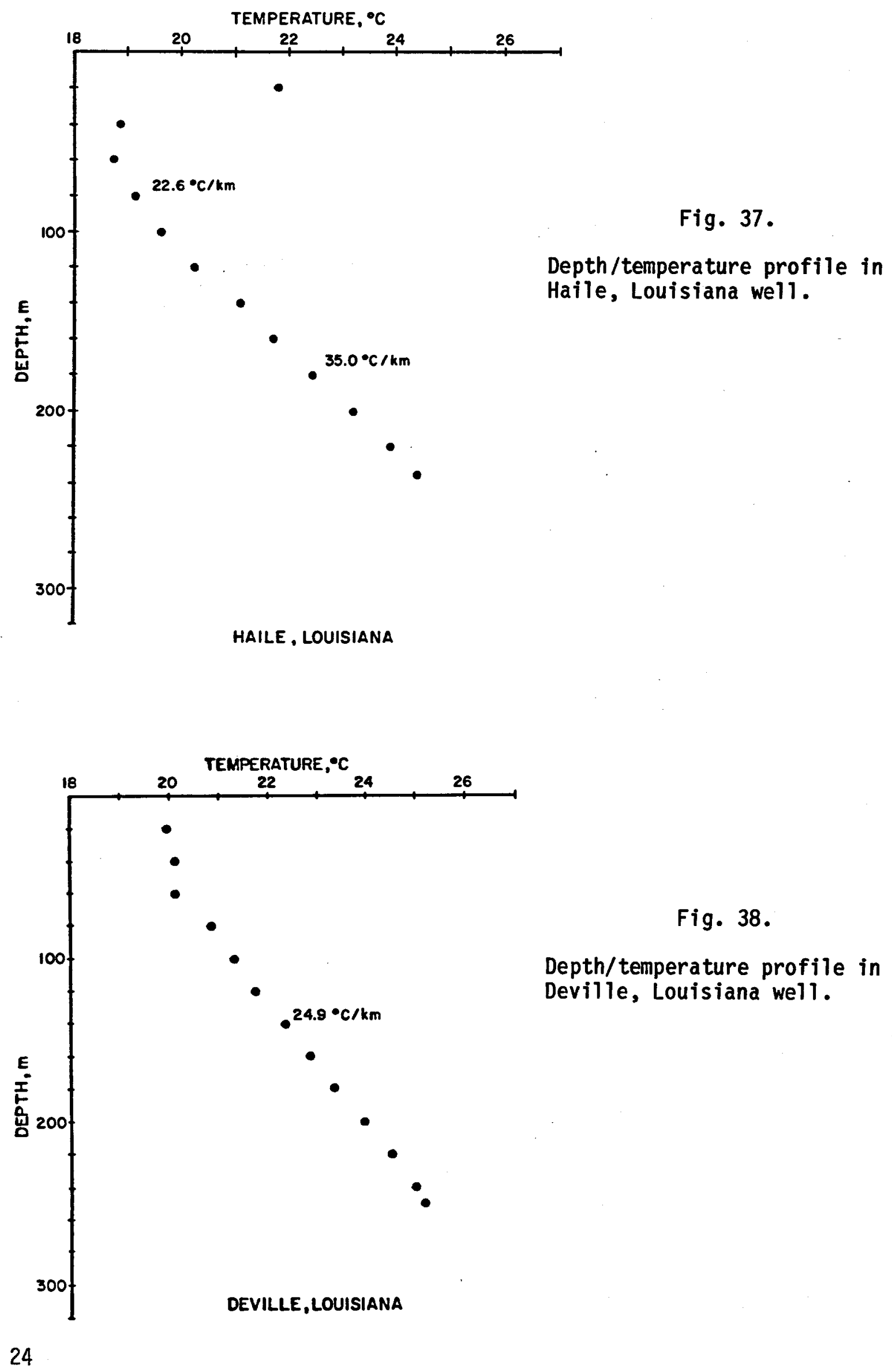

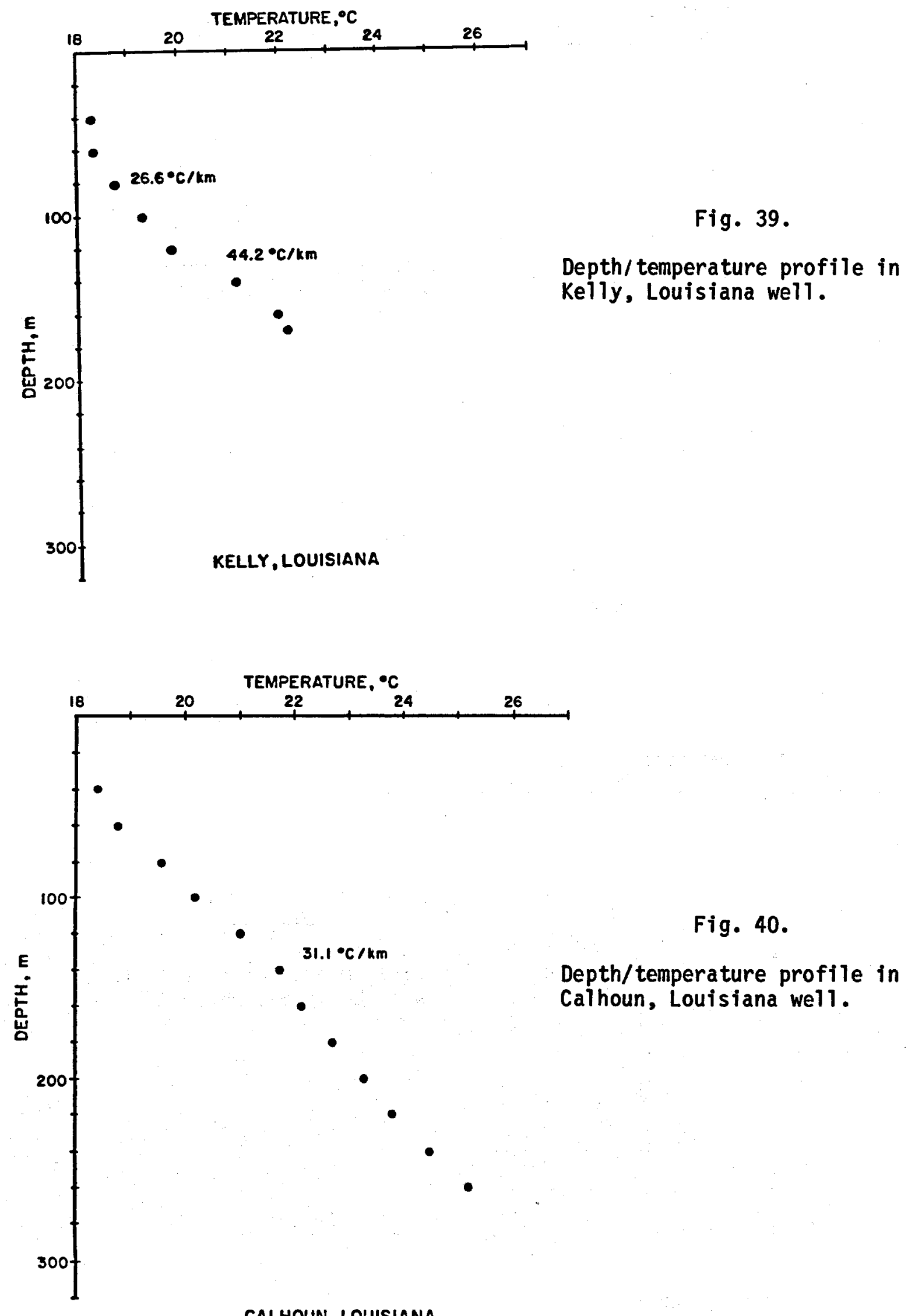

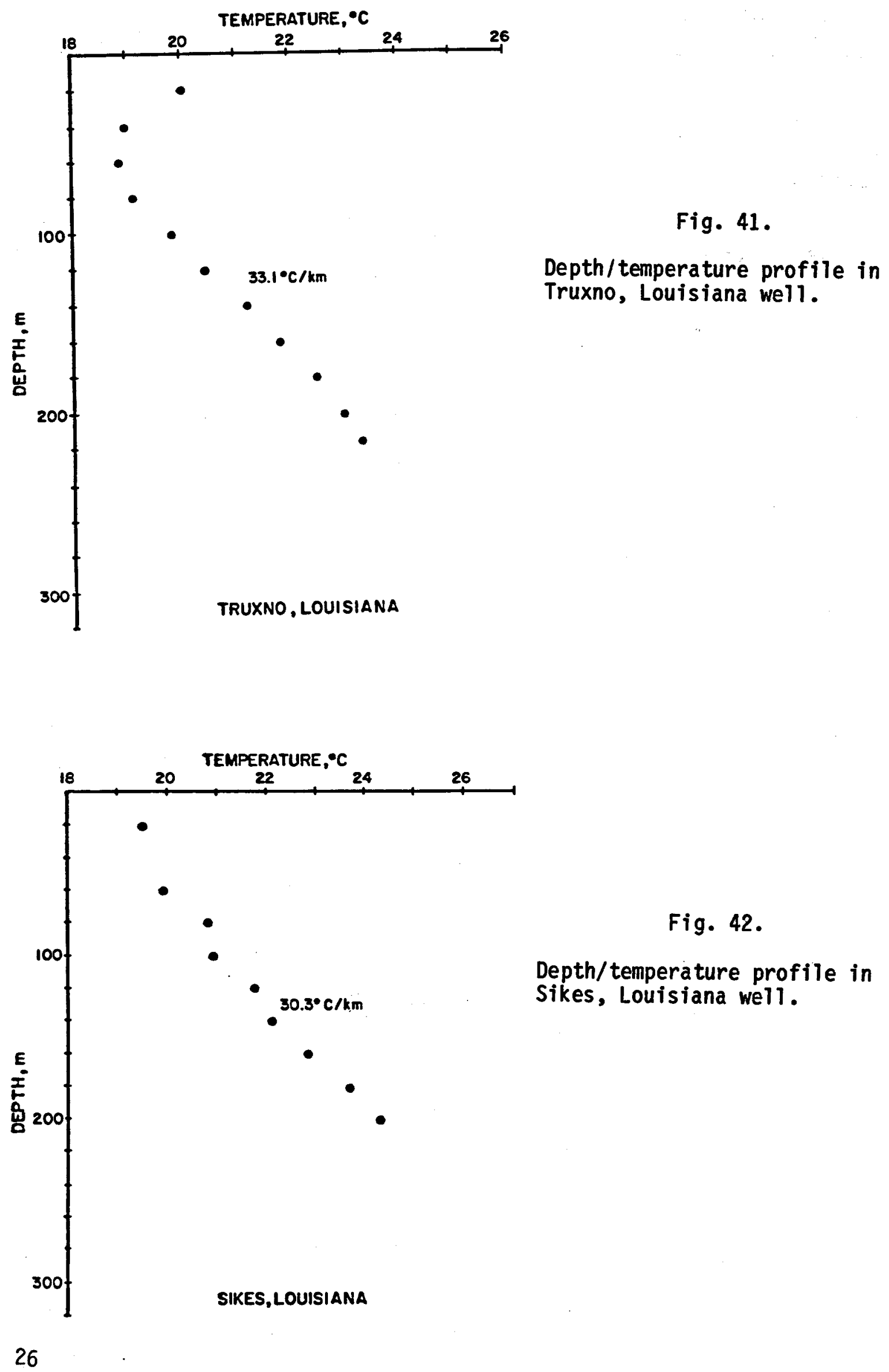

Fig. 42.

Depth/temperature profile in Sikes, Louisiana well. 


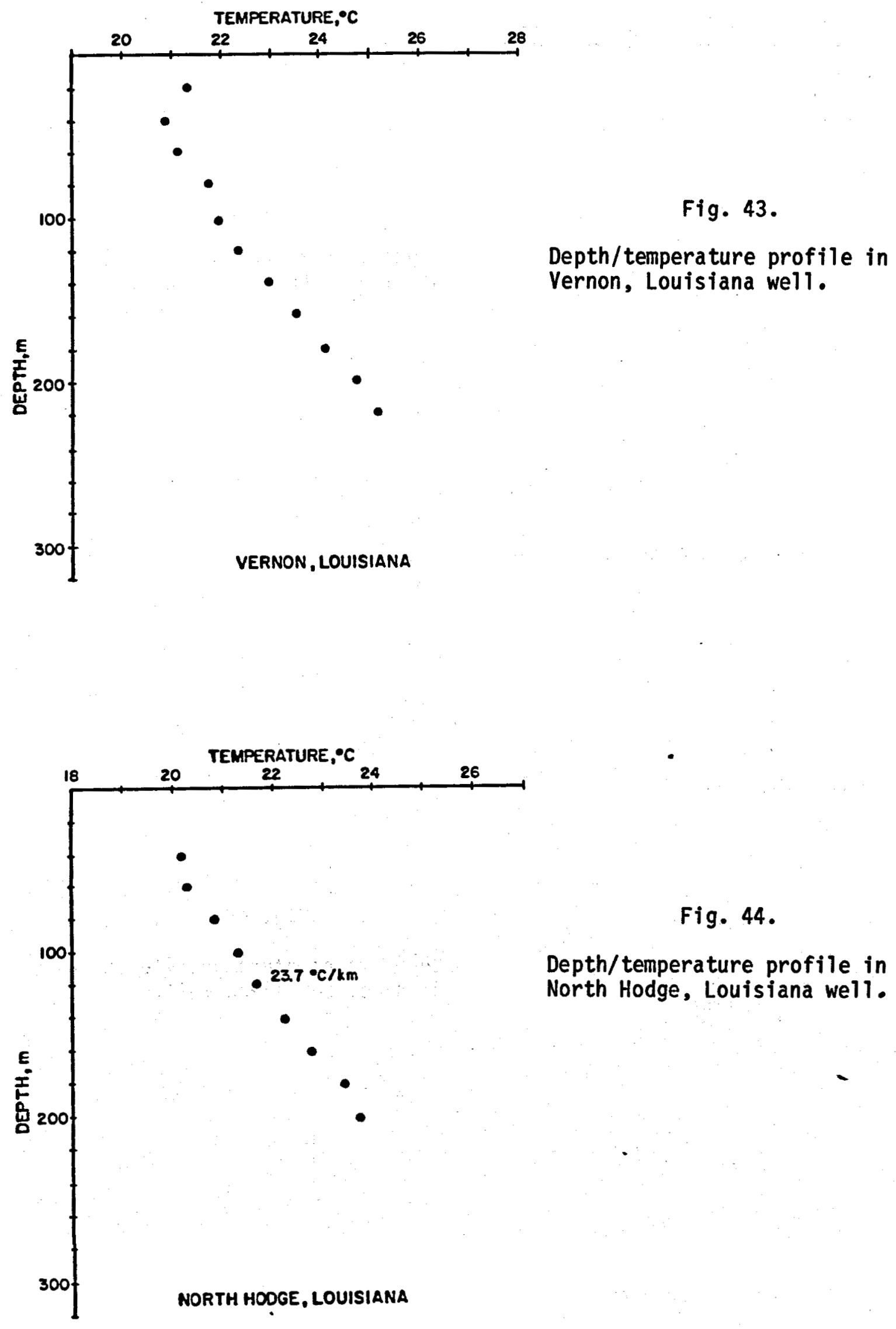




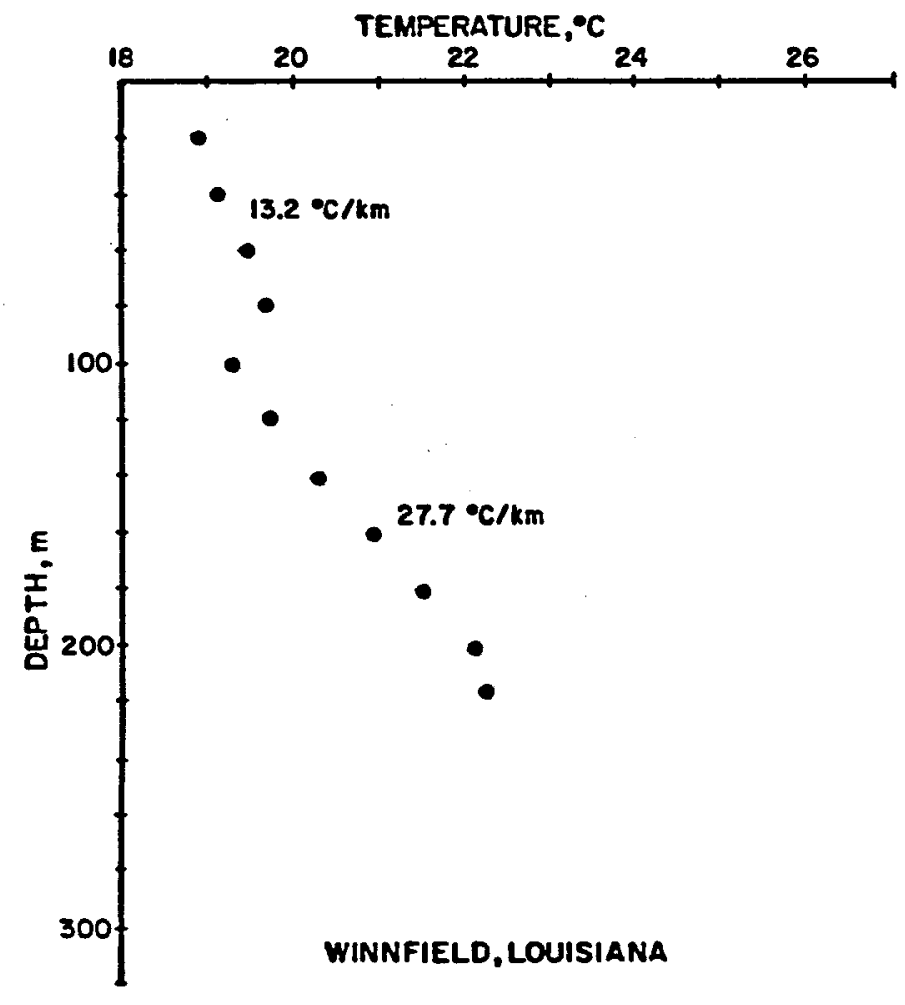

Fig. 45.

Depth/temperature profile in Winnfield, Louisiana well.

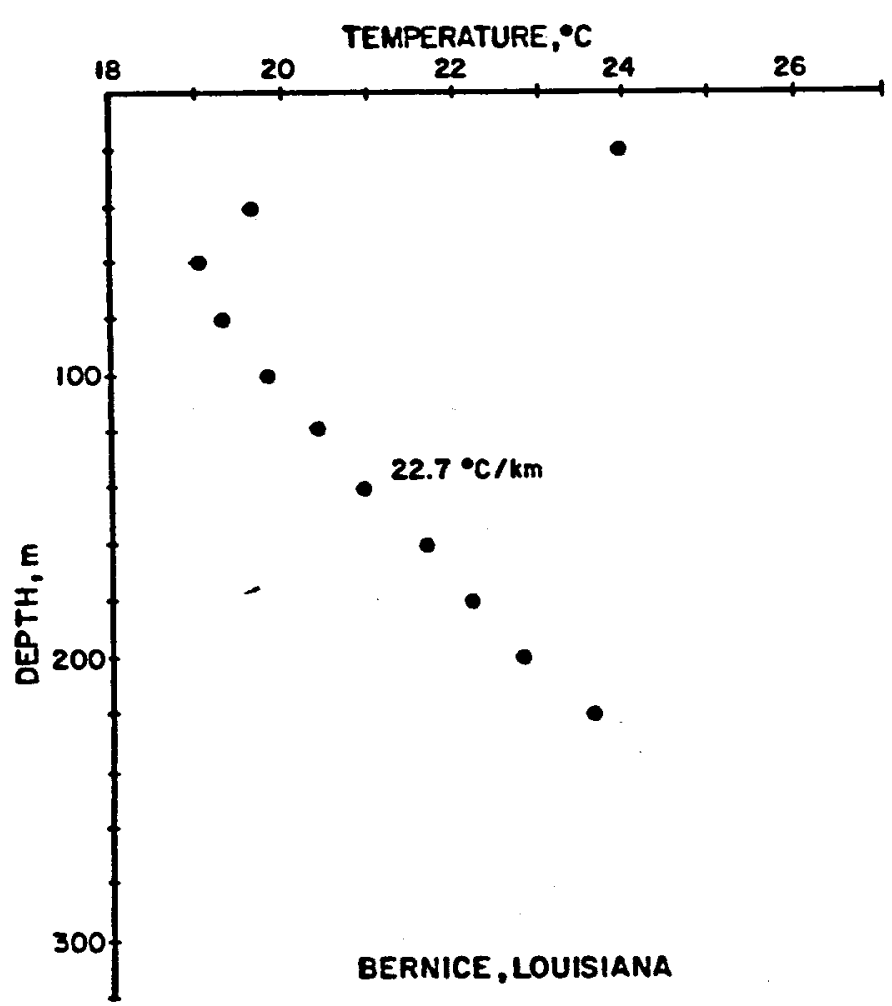

Fig. 46.

Depth/temperature profile in Bernice, Louisiana well. 


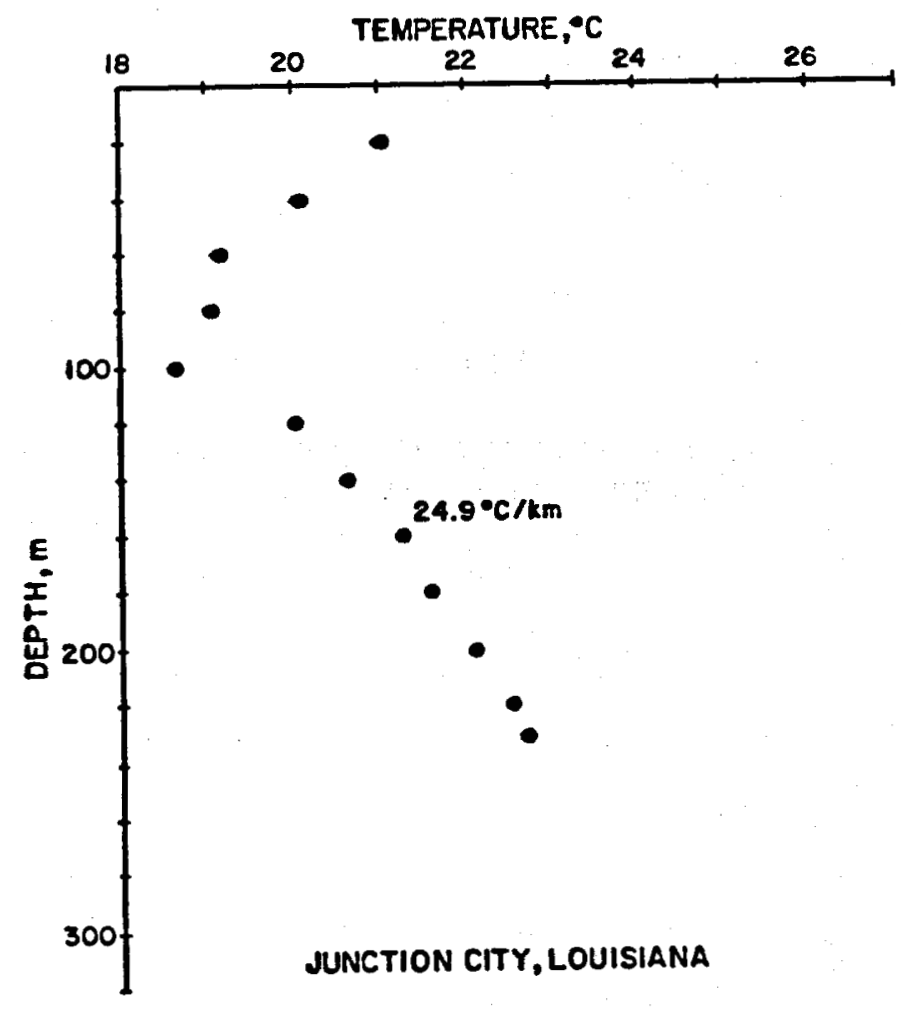

Fig. 47.

Depth/temperature profile in Junction City, Louisiana well.

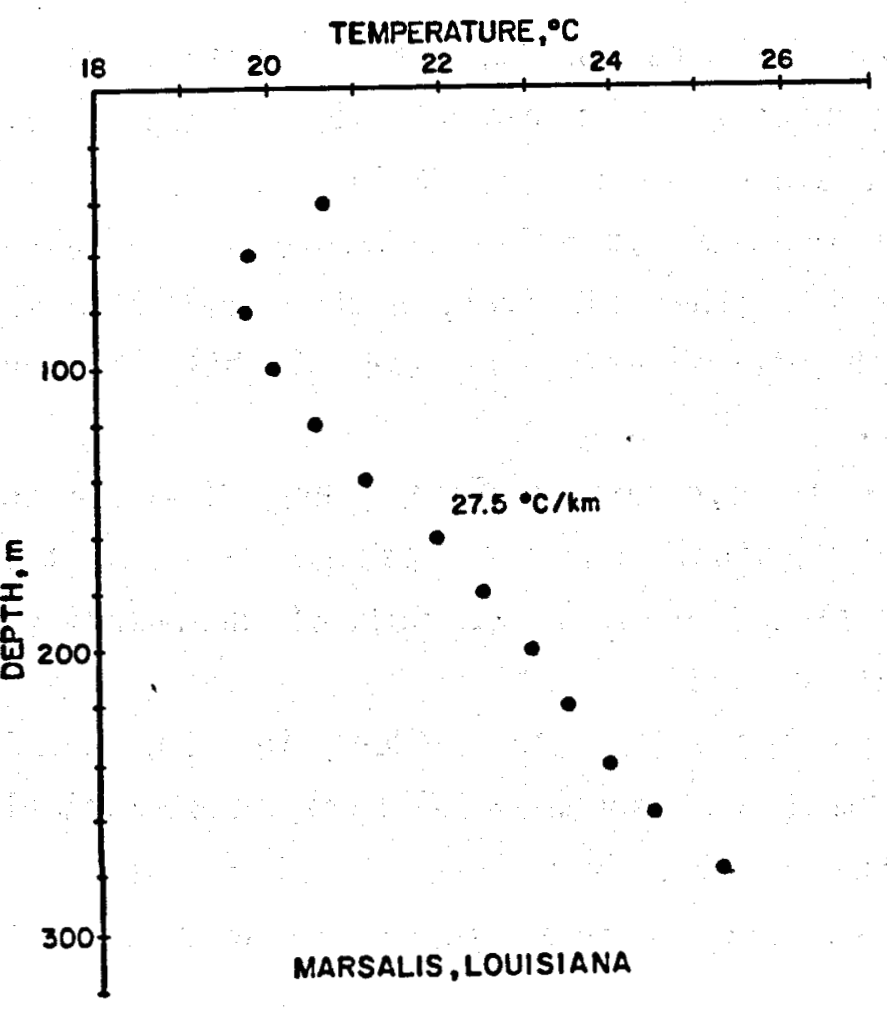

Fig. 48.

Depth/temperature profile in Marsal is, Louisiana well. 


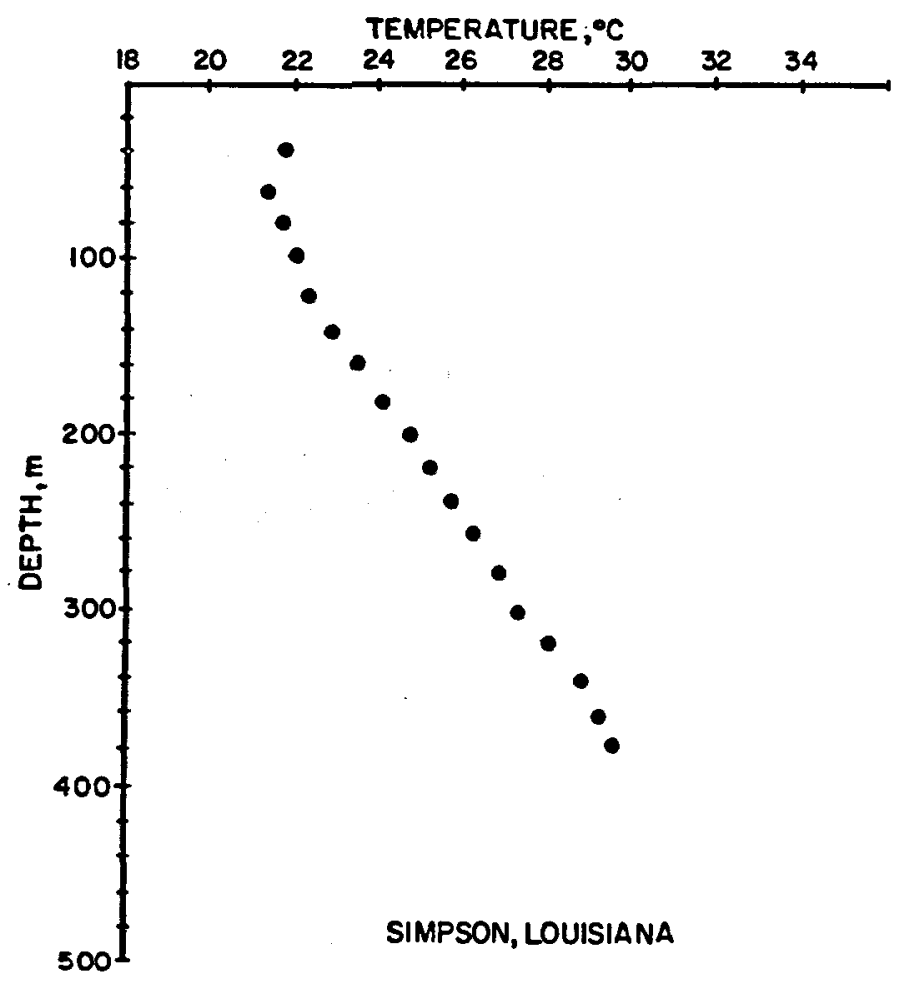

Fig. 49.

Depth/temperature profile in Simpson, Louisiana well.

Mississippi average $1.1 \mathrm{HFU}$, and thus approximate typical Gulf Coastal Plain values.

Quachita, Union, and Claiborne parishes in northern Louisiana where three sites exceed $2.0 \mathrm{HFU}$ and another six sites exceed $1.5 \mathrm{HFU}$ (Fig. 3 ). Geothermal gradients in northern Louisiana range from $25^{\circ} \mathrm{C} / \mathrm{km}$ to more than $40^{\circ} \mathrm{C} / \mathrm{km}$ in the $120-$ to $170-\mathrm{m}$ depth interval at Kelly. Several sites have gradients that exceed $30^{\circ} \mathrm{C} / \mathrm{km}$ (Table I). The relatively higher heat flow in northern Louisiana results from both higher gradients and slightly higher thermal-conductivity values (Table I).

The positive thermal anomalies in northern Louisiana are linked with higher gradients in west-central Mississippi and the western part of the Florida panhandle to form a wedge-shaped zone (Fig. 50) of near-surface thermal conditions in the gulf coastal area.

Positive and negative heat-flow anomalies are evident in Fig. 3 . A broad region, including most of central and southern Alabama, east-central Misissippi, southwestern Georgia, and north-central florida, has heat-flow values less than $0.7 \mathrm{HFU}$. In Alabama and Florida, these measurements are 


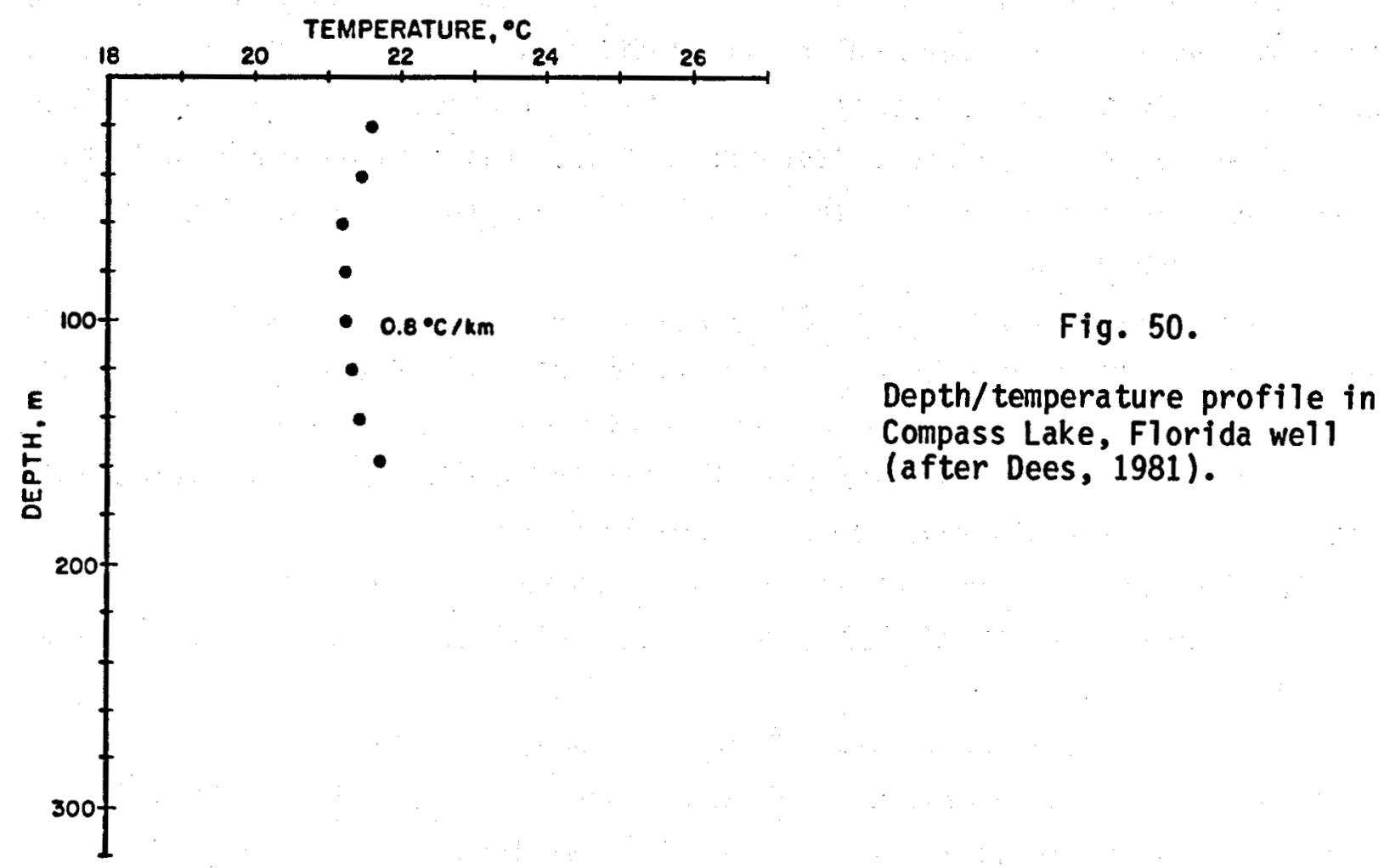

COMPASS LAKE, FLORIDA

consistent with earlier measurements in sedimentary rocks reported by Smith, Gregory, and Emhof (1981). The abnormally low heat-flow values can be attributed to exceptionally low geothermal gradients (typically $10-15^{\circ} \mathrm{C} / \mathrm{km}$ ). Only at Thomaston, Alabama, is a high geothermal gradient $\left(37^{\circ} \mathrm{C} / \mathrm{km}\right.$ over 140 to 280-m depth interval) associated with a low heat flux $10.9 \mathrm{HFU}$ in the same range; $0.7 \mathrm{HFU}$ weighted average).

The influence of hydrologic conditions is unclear although there may be some causal effects. Temperature measurements at Compass Lake, in the middle of the Florida panhandle, show a near-zero thermal gradient over a 160-m depth interval. As the top of the Florida aquifer is only $50 \mathrm{~m}$ deep there, a relation between the aquifer and the subsurface-temperature distribution is probable.

\section{DISCUSSION}

As Costain et a1. (1980) postulated $150^{\circ} \mathrm{C}$ temperatures at $2-\mathrm{km}$ depth, comparable extrapolations of certain temperature gradients in similar geologic 
environments in the Gulf Coastal Plain are proposed. Subsurface temperature extrapolations to depth, particularly in sedimentary terrains having extensive hydrologic activity, are conjectural. For the Gulf Coastal Plain, near-surface (0- to 500-m depth) temperature data can be considered with bottom-hole temperatures from deeper $0 i 1$ and gas tests to be of some use in predicting temperatures at greater depths.

Using general gradients of $25^{\circ} \mathrm{C} / \mathrm{km}$ and temperatures of $24^{\circ} \mathrm{C}$ at a depth of $200 \mathrm{~m}$ in northern Louisiana, a temperature range of $44^{\circ} \mathrm{C}$ might be expected at 1-km depth, and temperatures as high as $78^{\circ} \mathrm{C}$ can be postulated at $2 \mathrm{~km}$. If some perhaps anomalous gradients $\left(35-44^{\circ} \mathrm{C} / \mathrm{km}\right)$ are used, the extrapolated temperature values at depth would be considerably higher.

In the Pensacola area of the Florida panhandle, gradients of $30-34^{\circ} \mathrm{C} / \mathrm{km}$ and temperatures of $35^{\circ} \mathrm{C}$ at $500-\mathrm{m}$ depth can be extrapolated to $50^{\circ} \mathrm{C}$ temperatures at $1 \mathrm{~km}$.

Although definitions vary, low-grade geothermal resources that have potential use in space-heating and auxiliary-power for the eastern United States require temperatures of at least $40^{\circ} \mathrm{C}$ at less than $1-\mathrm{km}$ depth. Practical utilization of 1 ow-temperature $\left(<90^{\circ} \mathrm{C}\right)$ resources for direct-heat or heat-pump applications is also dependent on nearby consumer markets.

The near-surface temperature gradients observed in this study indicate that specific regions in northern Louisiana, western Mississippi, and the western part of the Florida panhandle may have the minimum geothermal conditions for eventual exploitation. The entire wedge-shaped zone described in Fig. 51 may contain marginal thermal resources.

In northern Louisiana a possible association between higher thermal conditions and salt intrusions can be inferred from Fig. 51. However, the area of known salt intrusions (Fig. 52) excludes several sites having higher heat-flow values in northern Louisiana, so it can be argued that the higher thermal conditions in northern Louisiana are spatially related to the distribution of subsurface alkalic plutons peripheral to the Monroe uplift. The relation of salt intrusions to high thermal gradients is of interest because the thermal conductivity of salt is three to four times greater than that of the country rock. Therefore, a salt diapir essentially would act as a preferential thermal conduit (Jacoby and Paul, 1974).

Smith, Dees, and Harrelson (1981) describe the northwesterly trending zone of anomalously low heat flow in southern Alabama and north-central 


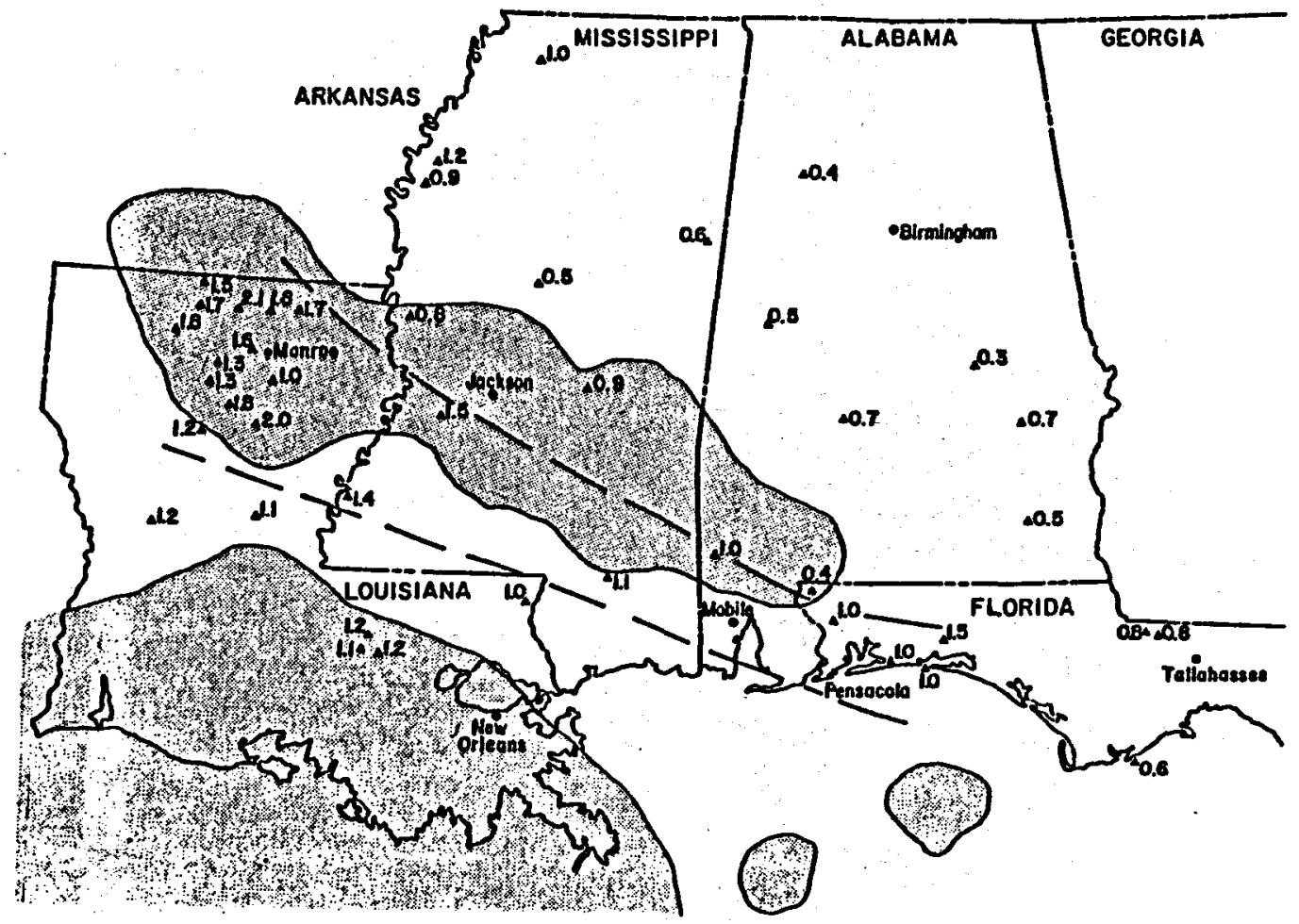

Fig. 51 .

Distribution of new heat-flow values in the Gulf coastal Plain. Shaded areas are those having salt-dome intrusions. The two dashed lines depict the zone of relatively high geothermal gradients (after Smith and Dees, in press).

Mississippi as being underlain by oceanic basement and a thick sequence of sedimentary rocks. The Pickens-Gilbertown fault system (Fig. 53) divides this zone on the south from oceanic crust distinguished by mobilized, thermally conductive salt diapirs. Smith, Dees, and Harrelson (1981) describe the Wiggins Arch intruded by Paleozoic granitic rocks in southernmost Mississippi and the Angelina-Caldwell flexure in southern Louisiana as representative of continental crust having average heat-flow values of 1.0-1.1 HFU.

Most drill holes in the Gulf Coastal Plain used in this reconnaissance penetrated one or more aquifers. The thermal influence of an aquifer commonly is perceptible on a depth/temperature profile. Most variations appear as discrete temperature offsets, whereas the temperature gradient may remain undisturbed or only sightly perturbed. Ideally, an aquifer having the capacity for convective overturn would act as a heat sink at its base and a heat source at its top, resulting in the predominance of convective rather than conductive heat transport. Such aquifers commonly have a relatively uniform internal 


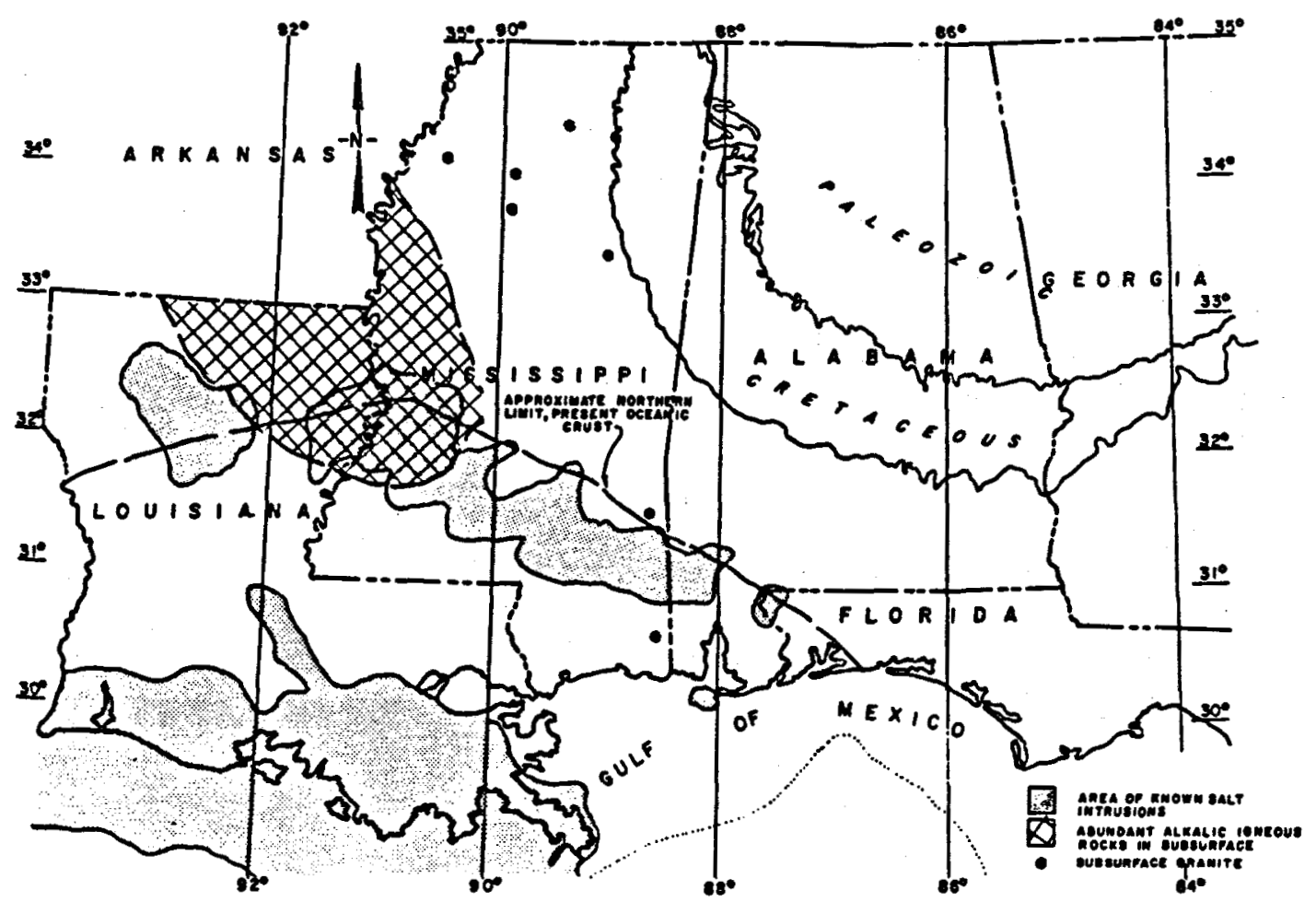

Fig. 52 .

Distribution of known subsurface igneous rocks and salt intrusions in the Gulf Coastal Plain (after Smith, Dees, and Harrelson, 1981).

temperature and a temperature gradient near zero. Such conditions may explain the exceptionally low temperature gradients and low heat-flow values determined in Alabama and north-central Florida. Throughout most of Mississippi and Louisiana, however, evidence of hydrologic influence on the thermal regime is less apparent.

\section{CONCLUSIONS}

A total of 46 new heat-flow values were determined for drill holes in the Gulf Coastal Plain of southwestern Georgia, Florida, Alabama, Mississippi, and Louisiana during the 20-month project. In general, the Gulf Coastal Plain is characterized by low-to-normal heat-flow values as well as exceptionally low temperature gradients in Alabama and peninsular Florida.

In extreme western Florida, thermal gradients to 500-m depth are approximately $30-34^{\circ} \mathrm{C} / \mathrm{km}$, and temperatures of $50^{\circ} \mathrm{C}$ can be extrapolated to $1 \mathrm{~km}$. 


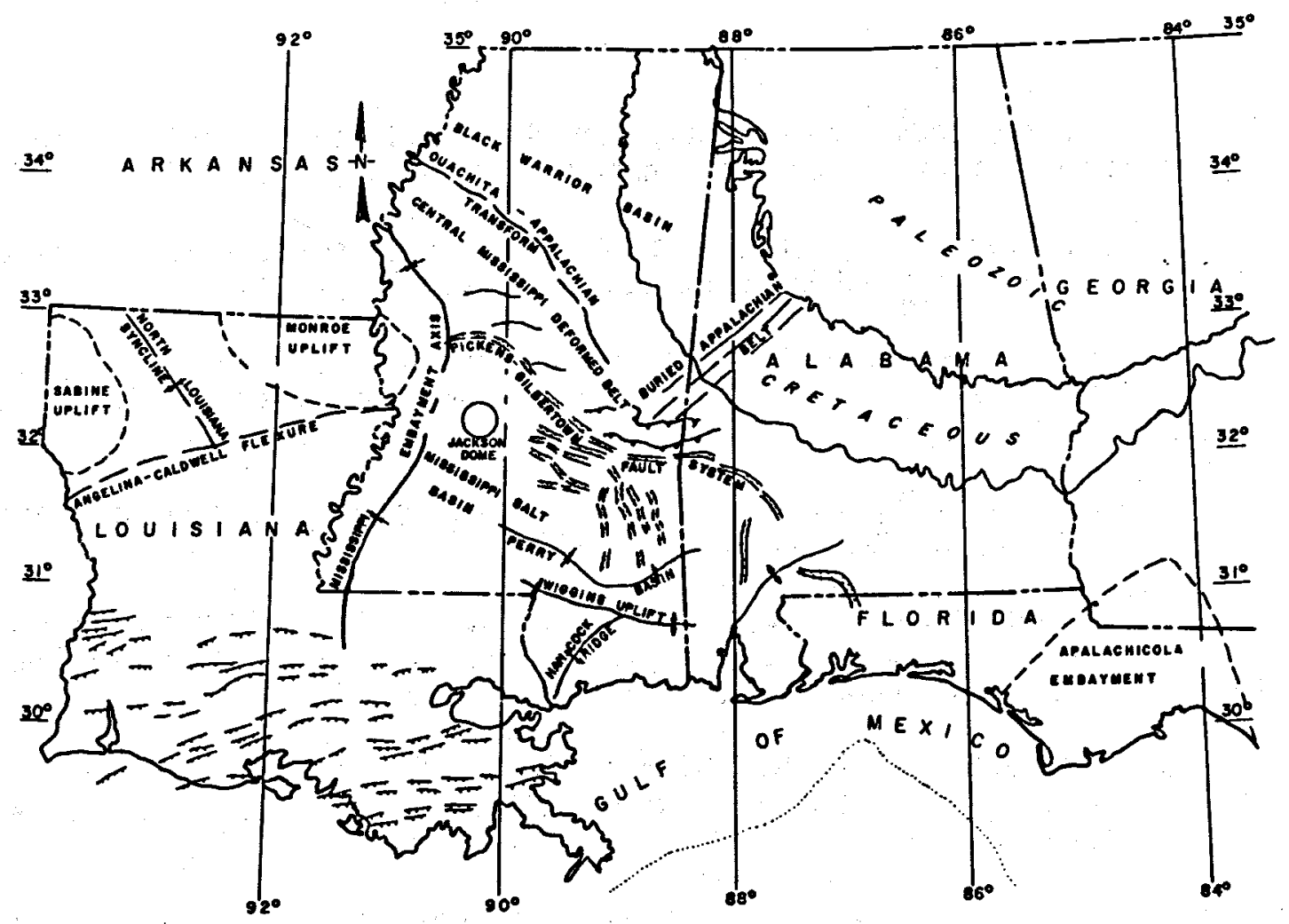

Fig. 53.

Subsurface structural features in the Gulf Coastal Plain (after Smith, Dees, and Harrison, 1981).

Continued evaluation of conditions in this area seems warranted in view of the substantial population and the industrial complex in the Pensacola-Mobile area.

In northern Louisiana (and a small but undetermined portion of southwestern Mississippi), thermal gradients of $35-46^{\circ} \mathrm{C} / \mathrm{km}$ and heat-flow values as large as 2.1 HFU are observed. A broad area of relatively high heat flow has been identified, and a three-parish area of particular interest has been recognized.

The near-surface thermal conditions observed to date in the Gulf Coastal plain do not warrant immediate development, but conditions in specific regions eventually may be considered for exploitation. 


\section{REFERENCES}

Beck, A., 1957, A steady-state method for the rapid measurement of the thermal conductivity of rocks, J. of Sci. Instrum., v. 34., pp. 86-189.

Costain, J. K., Glover, L., and Sinha, A. K., 1980, Low-temperature geothermal resources in the eastern United States, EOS, v. 61, pp. 1-3.

Dees, W. T., 1981, Heat flow, basement relationships, and geothermal prospects in the southeastern Gulf coast province, Univ. of Florida $M$. $S$. thesis, $116 \mathrm{p}$.

Jacoby, C. H., and Paul, D. K., 1974, Salt domes as a source of geothermal energy, Min. Eng., v. 26, no. 5, pp. 34-39.

Sass, J. H., Lachenbruch, A. H., and Munroe, R. J., 1971, Thermal conductivity of rocks from measurements on fragments and its application to heat flow determinations, J. of Geophys. Research, v. 76, pp. 3391-3401.

Sass, J. H., Lachenbrach, A. H., Munroe, R. J., Greene, G. W., and Moses, T. H., 1971, Heat flow in the western United States, J. of Geophys. Res., $v$. 76, pp. 6376-6413.

Smith, D. L. and Dees, W. T., in press, Indicators of low temperature geothermal resources in northern Louisiana and central Mississippi, $J$. Volcanology and Geothermal Res., 3 p.

Smith, D. L., and Dees, W. T., 1982, Heat flow in the Gulf Coast region, J. of Geophys. Res.

Smith, D. L., Dees, W. T., and Harrelson, D. W., 1981, Geothermal conditions and their implications for basement tectonics in the Gulf Coast margin, Gulf Coast Assoc. of Geol. Soc. Trans., 31st Ann. Meet., pp. 181-190.

Smith, D. L., Gregory, R. G., and Emhof, J. W., 1981, Geothermal measurements in the southern Appalachian Mountains and southeastern coastal plains, Am. J. of Sci., v. 281, pp. 282-298.

von Frese, R. R. B., Hinze, W. J., Braile, R. W., Swanberg, C. A., Morgan, P., Lidiak, E. G., Roy, R. S., Keller, G. R., Aiken, C., and Denison, R. E., 1980, Midcontinent HDR program, in "Geothermal Energy and the Eastern United States," Johns Hopkins University Applied Physics Laboratory report QM-80-185, Pp. VIII 1-19. 


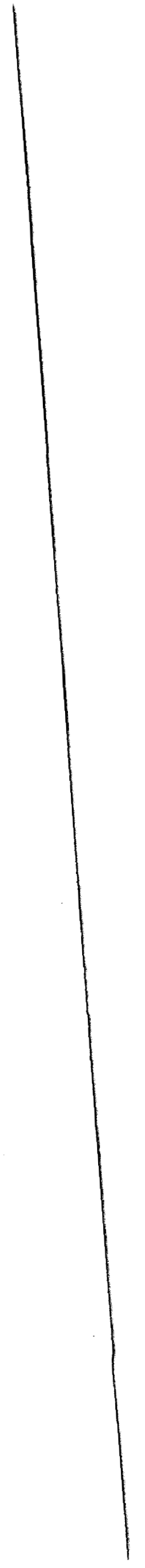




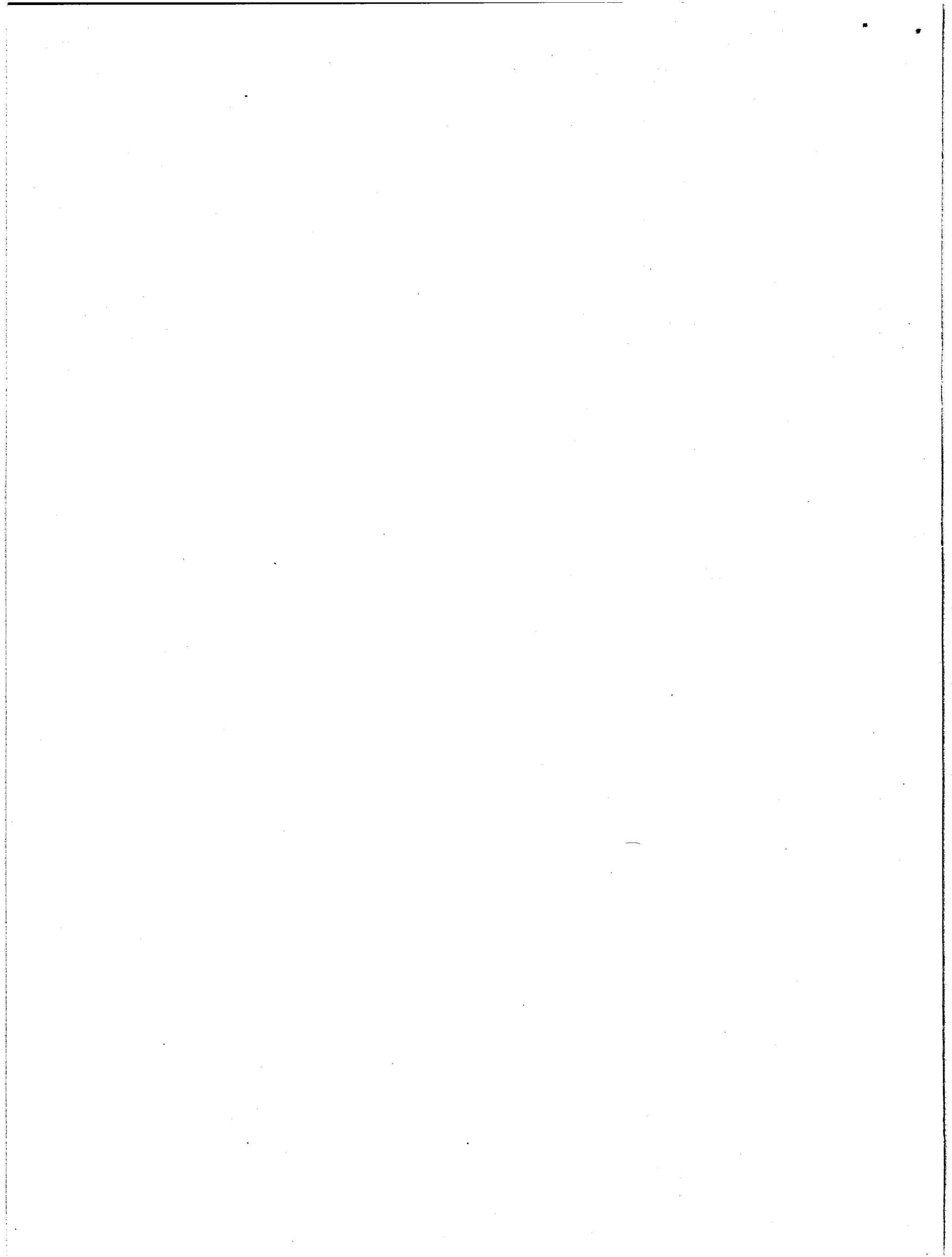

